\title{
Special Galileon at one loop
}

\section{Filip Přeučil and Jiří Novotný}

Institute of Particle and Nuclear Physics, Faculty of Mathematics and Physics, Charles University,

V Holešovičkách 2, CZ-180 00 Prague 8, Czech Republic

E-mail: Preucil@ipnp.troja.mff.cuni.cz,

Novotny@ipnp.troja.mff.cuni.cz

Abstract: We present a complete one-loop renormalization of the Special Galileon $S$ matrix. Especially, we give a complete list of the higher derivative operators which are necessary for the one-loop on-shell renormalization and prove the invariance of the oneloop on-shell effective action with respect to the Special Galileon symmetry. This enables us to enlarge the validity of the enhanced $O\left(p^{3}\right)$ soft behavior of the scattering amplitudes to the one-loop level. As an illustration, we discuss explicitly the four-point and five-point one-loop scattering amplitudes and comment on some conjectures appearing in the existing literature.

Keywords: Effective Field Theories, Scattering Amplitudes

ArXIV EPRINT: 1909.06214 


\section{Contents}

1 Introduction: why is the Special Galileon special? $\quad 1$

2 The generating functional of the $S$-matrix and the on-shell effective action

3 Quantum fluctuations in the classical background

4 The UV divergences at one loop

5 The scattering amplitudes at one loop and the soft theorem

6 Special Galileon amplitudes at one loop - explicit example

7 Summary and discussion

\section{Introduction: why is the Special Galileon special?}

The Galileons are remarkable derivatively coupled scalar effective field theories with a plethora of very interesting properties both at the classical and the quantum level. In the literature, the (cubic) Galileon emerged for the first time as the only interacting (zero helicity) mode in the decoupling limit of the Dvali-Gababadze-Poratti modified gravity model $[1,2]$ and, almost at the same time, also in the similar limit of the massive gravity model [3]. Soon it has been recognized [4] that the Galileon in its generalized form might be a promising local modification of General relativity at large scales with several appealing properties. In particular, regardless of the presence of highly nonlinear higher derivative couplings in the Lagrangian, the Galileon obeys a second-order equation of motion which ensures the absence of the Ostrogradsky ghosts. It also has been shown that near the massive sources the Galileon field is suppressed by the Vainshtein screening mechanism [5], and that the basic Lagrangian is stable with respect to the quantum corrections [6-8]. The seminal paper [4] initiated a boom of increasing interest in the Galileon theories concerning their formal properties, the possible generalizations, e.g. $[9,10]$ as well as the cosmological applications. For a pedagogical review and for a more comprehensive list of the existing literature, see e.g. [11, 12].

In the general case, the basic flat space Galileon Lagrangian collects the most general terms built of the single scalar field and its derivatives (up to and including the second order ones with $n$ fields and $2 n-2$ derivatives). These terms can be uniquely determined 
by the requirement of yielding a second-order equation of motion and by invariance with respect to the polynomial shift symmetry of the first order,

$$
\delta \phi=a+b \cdot x,
$$

where $a$ and $b_{\mu}$ are real parameters. The latter property has an important consequence at the quantum level, namely the tree-level on-shell scattering amplitudes pose an enhanced soft behavior [13-15]. This means that the scattering amplitudes vanish as a second power of momentum when one of the external particles becomes soft, which is a higher power than one would naively expect from a simple counting of the derivatives in the Lagrangian. The general basic Galileon Lagrangian in $D$ dimensions can be written in the form

$$
\mathcal{L}_{\mathrm{b}}=\sum_{n=0}^{D} d_{n+1} \phi \varepsilon^{\mu_{1} \ldots \mu_{D}} \varepsilon^{\nu_{1} \ldots \nu_{D}} \prod_{i=1}^{n} \partial_{\mu_{i}} \partial_{\nu_{i}} \phi \prod_{j=n+1}^{D} \eta_{\mu_{j} \nu_{j}}
$$

where $d_{n}$ are free real $n$-point couplings. For the theory to be well defined on the quantum level, we demand $d_{1}=0$ (no tadpoles) and $d_{2}=(-1)^{D} / 2(D-1)$ ! (canonical normalization of the kinetic term). The above mentioned enhanced soft behavior enables the full on-shell reconstructibility of the tree-level scattering amplitudes once the basic set of the seed amplitudes from the four-point one up to the $(D+1)$-point one is known [14]. On the other hand, due to the rich set of dualities of the Galileon Lagrangian [16-19], there is a many-to-one correspondence between the constants $d_{n}$ and the on-shell physics represented by the scattering amplitudes.

The particular choice of the couplings $d_{n}$ can further increase the symmetry of the Lagrangian (1.2). Namely, we mean the choices

$$
d_{2 n}^{(+)}=\frac{(-1)^{n}}{2 n}\left(\begin{array}{c}
D \\
2 n-1
\end{array}\right) \frac{\cos \beta}{\alpha^{2(n-1)}}, \quad d_{2 n+1}^{(+)}=\frac{(-1)^{n}}{2 n+1}\left(\begin{array}{c}
D \\
2 n
\end{array}\right) \frac{\sin \beta}{\alpha^{2 n-1}},
$$

or

$$
d_{2 n}^{(-)}=\frac{1}{2 n}\left(\begin{array}{c}
D \\
2 n-1
\end{array}\right) \frac{\cosh \beta}{\alpha^{2(n-1)}}, \quad d_{2 n+1}^{(-)}=\frac{1}{2 n+1}\left(\begin{array}{c}
D \\
2 n
\end{array}\right) \frac{\sinh \beta}{\alpha^{2 n-1}}
$$

Here $\alpha$ has the dimension [mass] ${ }^{(D+2) / 2}$ and $\alpha^{2 /(D+2)}$ is therefore the scale which controls the size of nonlinearities in the Lagrangian (1.2), while $\beta$ is a dimensionless parameter. The prescription (1.3) and (1.4) yields two branches of two-parametric families of Lagrangians $\mathcal{L}^{( \pm)}(\alpha, \beta)$ which are invariant with respect to the generalized polynomial shift symmetry $[20,21]$

$$
\delta \phi=\frac{\theta}{2} H^{\mu \nu}\left(\alpha^{2} x_{\mu} x_{\nu} \pm \partial_{\mu} \phi \partial_{\nu} \phi\right),
$$

where $H^{\mu \nu}$ is an arbitrary fixed traceless ${ }^{1}$ symmetric tensor and $\theta$ is an infinitesimal parameter. On the quantum level, this symmetry is responsible for a further enhancement of the soft behavior. In the above theories with $\beta=0$, the tree-level amplitudes vanish

\footnotetext{
${ }^{1}$ For $H_{\mu}^{\mu} \neq 0$, the transformation (1.5) is a duality of the family which transforms the Lagrangian (1.2) and (1.4) with parameters $(\alpha, \beta)$ into the Lagrangian with parameters $\left(\alpha, \beta \mp \theta \alpha H_{\mu}^{\mu}\right)$.
} 
as the third power of momentum in the single particle soft limit. The latter theories (1.3) and (1.4) with $\beta=0$ are known as the Special Galileons [13, 22].

The Special Galileons are special in several aspects. First, their soft behavior is in a sense extremal since, as it has been shown in [15], the $O\left(p^{3}\right)$ single soft limit is the highest possible within the single scalar effective theories with a non-trivial power counting. To be more precise, let us assume a derivatively coupled single scalar effective field theory, and for each elementary vertex $V$ in the Lagrangian, let us determine the ratio

$$
\rho_{V}=\frac{D_{V}-2}{E_{V}-2}
$$

where $D_{V}$ is a number of derivatives and $E_{V}$ is a number of external legs of the vertex $V$. The soft behavior of such a theory can be characterized by the soft exponent $\sigma$ which corresponds to the $O\left(p^{\sigma}\right)$ behavior of the amplitudes in the single particle soft limit $p \rightarrow 0$. Then, for the general Galileon, we get $\rho_{V}=2$ for each admissible vertex, and the soft exponent is $\sigma=2$. Provided we assume only theories with a fixed $\rho_{V}=\rho \geq 1$, i.e. only with vertices with $2+\rho\left(E_{V}-2\right)$ derivatives and with the soft limit characterized by a soft exponent $\sigma$, then the theories with a non-trivial enhanced soft behavior are those for which $\rho \leq \sigma$ since in the opposite case, there is enough derivatives per field in each vertex to ensure the $O\left(p^{\sigma}\right)$ soft limit automatically. However, as shown in [15], not all the non-trivial pairs $(\rho, \sigma)$ are admissible, namely they have to satisfy the bounds $\rho \geq \sigma-1$ and $\sigma \leq 3$. Note that the Special Galileon saturate both these bounds, sitting in the very corner of the admissible $(\rho, \sigma)$ region. This means that the Special Galileon together with the Dirac-Born-Infeld theory and the Non-linear sigma model belongs to the set of the exceptional scalar effective field theories for which there exists the Cachazo-He-Yuan representation [22].

The second special property of the Special Galileon is that it is (contrary to the general Galileon theories) potentially well defined as an perturbative effective quantum field theory - at least formally when we assume that the regularization and renormalization preserves all the symmetries. The full Lagrangian is then

$$
\mathcal{L}=\mathcal{L}_{\mathrm{b}}+\mathcal{L}_{\mathrm{CT}}
$$

where $\mathcal{L}_{\mathrm{CT}}$ corresponds to the higher derivative counterterms needed for the perturbative renormalization. Here we tacitly assume that $\mathcal{L}_{\mathrm{CT}}$ shares the symmetries of the basic Lagrangian $\mathcal{L}_{b}$. As shown in [19], it is possible to organize the perturbative expansion according to the hierarchy of the vertices and according to the hierarchy of the corresponding Feynman graphs. This hierarchy is based on the assignment of an index $i_{V}$ to each vertex $V$ (including those coming from $\mathcal{L}_{\mathrm{CT}}$ ) where

$$
i_{V}=D_{V}-\left(2 E_{V}-2\right)
$$

Here $D_{V}$ and $E_{V}$ have the same meaning as above. Note that for the basic Lagrangian (1.2), we get $i_{V}=0$, and therefore this index measures the abundance of the number of derivatives relatively to the basic Lagrangian $\mathcal{L}_{\mathrm{b}}$. The index of the counterterm vertices which are 
necessary in order to renormalize the one particle irreducible graph $\Gamma$ is then given (for a derivation of this formula, see [19])

$$
i_{\mathrm{CT}}^{\Gamma}=(D+2) L_{\Gamma}+\sum_{V \in \Gamma} i_{V}
$$

where $L_{\Gamma}$ is the number of loops, and $i_{V}$ are the indices of the vertices of the graph $\Gamma$. The indices of the counterterms are thus related to the loop expansion. Therefore, in principle, the perturbative renormalization in the effective theory sense would be possible provided there is only a finite number of vertices with fixed $i_{\mathrm{CT}}$. Then at each level of the hierarchy, given by the contributions of all the Feynman graphs $\Gamma$ with $i_{\mathrm{CT}}^{\Gamma}$ fixed, there would be only a finite number of unknown parameters.

In the case of the general Galileon, the only other constraint for the counterterms is the invariance with respect to the symmetry transformation (1.1). Then, however, there is an infinite number of possible uncorrelated vertices with given fixed $i_{\mathrm{CT}}$, and therefore the formal effective theory loses its predictivity at each hierarchy level (see [19] for a more detailed discussion).

On the other hand, the generalized polynomial shift symmetry (1.5) of the Special Galileon is strong enough to restrict the form of the counterterm Lagrangian with $i_{\mathrm{CT}}$ fixed in such a way that at each hierarchy level, there is only a finite number of unknown parameters in the $S$-matrix. The situation here is somewhat similar to the case of chiral perturbation theory $[23,24]$, where the nonlinearly realized chiral symmetry allows only for a finite number of low energy couplings at each order in the derivative expansion. This of course does not mean that there is only a finite number of vertices at each hierarchy level. Rather the infinite number of possible vertices have correlated couplings, and as a result, they are combined into a finite number of operators invariant with respect to (1.5).

The third distinguished feature of the Special Galileon is the beautiful geometry behind the polynomial shift symmetry (1.5). The Special Galileon field can be understood as a scalar degree of freedom which describes fluctuations of a $D$-dimensional brane in a $2 D$-dimensional pseudo-Riemannian target space $\mathbb{R}^{2,2 D-2}$ treated as a Kähler manifold. ${ }^{2}$ The hidden Special Galileon symmetry (1.5) corresponds then to the nonlinearly realized subgroup of the symmetry group $\mathbb{C}^{D} \rtimes U(1, D-1)$ of the target space. This interpretation of the Special Galileon allows for a simple construction and classification of the counterterm Lagrangian $\mathcal{L}_{\mathrm{CT}}$ (see [21] for more details). ${ }^{3}$ The basic building blocks for higher derivative Lagrangians are then the effective metric (the \pm in the following formulas correspond to the two choices of the transformation prescription (1.5))

$$
g_{\mu \nu}=\eta_{\mu \nu} \pm \frac{1}{\alpha^{2}} \partial_{\mu} \partial \phi \cdot \partial \partial_{\nu} \phi
$$

the extrinsic curvature tensor

$$
\mathcal{K}_{\alpha \mu \nu}=-\frac{1}{\alpha} \partial_{\alpha} \partial_{\mu} \partial_{\nu} \phi
$$

\footnotetext{
${ }^{2}$ This is the case of the "plus" branch of the Special Galileon. For the "minus" branch, an appropriate analytic continuation of the parameter $\alpha$ is necessary. The target space is then $\mathbb{R}^{D, D}$, which has no compatible complex structure. See [21] for more details.

${ }^{3}$ An alternative way of classification of higher derivative Special Galileon Lagrangians has been developed in $[25,26]$ using the coset construction.
} 
the Christoffel symbol

$$
\Gamma_{\rho \sigma \mu}= \pm \frac{1}{\alpha^{2}} \partial_{\mu} \partial_{\sigma} \partial \phi \cdot \partial \partial_{\rho} \phi
$$

the corresponding covariant derivative $D_{\mu}$, and the covariant Levi-Civita tensor

$$
E^{\mu_{1} \ldots \mu_{D}}=\frac{1}{\sqrt{g}} \varepsilon^{\mu_{1} \ldots \mu_{D}},
$$

where $g$ is the absolute value of the determinant of the effective metric $g_{\mu \nu}$. Any diffeomorphism invariant built from $g_{\mu \nu}$ and its inverse $g^{\mu \nu}, \mathcal{K}_{\alpha \mu \nu}$, and their covariant derivatives ${ }^{4}$ is automatically invariant with respect to (1.5). In order to construct the action, we need also the invariant measures, namely, for the plus sign in (1.5), the measures $\mathrm{d}^{D} Z$ and $\mathrm{d}^{D} \bar{Z}$

$$
\mathrm{d}^{D} Z=\mathrm{d}^{D} x \operatorname{det}\left(\eta+\frac{\mathrm{i}}{\alpha} \partial \partial \phi\right), \quad \mathrm{d}^{D} \bar{Z}=\mathrm{d}^{D} x \operatorname{det}\left(\eta-\frac{\mathrm{i}}{\alpha} \partial \partial \phi\right)
$$

or, for the minus sign in (1.5), the measures $\mathrm{d}^{D} Z^{+}$and $\mathrm{d}^{D} Z^{-}$

$$
\mathrm{d}^{D} Z^{ \pm}=\mathrm{d}^{D} x \operatorname{det}\left(\eta \pm \frac{1}{\alpha} \partial \partial \phi\right)
$$

and the canonical one

$$
\mathrm{d}^{D} x \sqrt{g}=\left\{\begin{array}{c}
\sqrt{\mathrm{d}^{D} Z \mathrm{~d}^{D} \bar{Z}} \\
\sqrt{\mathrm{d}^{D} Z^{+} \mathrm{d}^{D} Z^{-}}
\end{array} .\right.
$$

As discussed in [21], the typical off-shell counterterm action which is invariant with respect to the generalized polynomial shift symmetry (1.5) is e.g. of the form

$$
S_{\mathrm{CT}}^{(+)}=\int \sqrt{\mathrm{d}^{D} Z \mathrm{~d}^{D} \bar{Z}} \mathcal{L}_{Z \bar{Z}}+\int \mathrm{d}^{D} Z \mathcal{L}_{Z}+\int \mathrm{d}^{D} \bar{Z} \mathcal{L}_{\bar{Z}}
$$

where $\mathcal{L}_{Z}^{*}=\mathcal{L}_{\bar{Z}}$, or

$$
S_{\mathrm{CT}}^{(-)}=\int \sqrt{\mathrm{d}^{D} Z^{+} \mathrm{d}^{D} Z^{-}} \mathcal{L}_{ \pm}+\int \mathrm{d}^{D} Z^{+} \mathcal{L}_{+}+\int \mathrm{d}^{D} Z^{-} \mathcal{L}_{-}
$$

according to the sign in (1.5) and in (1.9). The functions $\mathcal{L}_{Z \bar{Z}}, \cdots, \mathcal{L}_{-}$are diffeomorphism invariants built from the above geometrical building blocks.

However, this is not the whole story. As a consequence of the invariance of the independent measures $\mathrm{d}^{D} Z^{ \pm}$, or $\mathrm{d}^{D} Z$ and $\mathrm{d}^{D} \bar{Z}$, we can construct another invariant given as

$$
\sigma=\frac{\alpha}{2 \mathrm{i}} \ln \left[\frac{\operatorname{det}\left(\eta+\frac{\mathrm{i}}{\alpha} \partial \partial \phi\right)}{\operatorname{det}\left(\eta-\frac{\mathrm{i}}{\alpha} \partial \partial \phi\right)}\right],
$$

for the "plus" branch of the transformation (1.5) and

$$
\sigma=\frac{\alpha}{2} \ln \left[\frac{\operatorname{det}\left(\eta+\frac{1}{\alpha} \partial \partial \phi\right)}{\operatorname{det}\left(\eta-\frac{1}{\alpha} \partial \partial \phi\right)}\right],
$$

\footnotetext{
${ }^{4}$ Thanks to the relations between these geometrical objects, the other building blocks, e.g. the Riemann tensor and its descendants, are not needed without any loss of generality.
} 
for the "minus" branch. Note that these invariants were missed in [21]. The existence of these invariants allows to forget the measures $\mathrm{d}^{D} Z$ and $\mathrm{d}^{D} \bar{Z}$, or $\mathrm{d}^{D} Z^{+}$and $\mathrm{d}^{D} Z^{-}$ since these can be constructed using the canonical measure (1.15) and the functions of the invariants $\sigma$, e.g.

$$
\mathrm{d}^{D} Z=\mathrm{d}^{D} x \sqrt{g} \mathrm{e}^{\frac{\mathrm{i}}{\alpha} \sigma} .
$$

The most general off-shell counterterm action is then for the two branches of the Special Galileon

$$
S_{\mathrm{CT}}=\int \mathrm{d}^{D} x \sqrt{g} \mathcal{L}_{\mathrm{CT}}\left(\sigma, D \sigma, \ldots, \mathcal{K}, D \mathcal{K}, \ldots, g^{\mu \nu}\right),
$$

where $\mathcal{L}_{\mathrm{CT}}$ is a diffeomorphism invariant built from the scalar $\sigma$, the extrinsic curvature tensor $\mathcal{K}_{\mu \nu \alpha}$, their covariant derivatives, the inverse metric $g^{\mu \nu}$, and the Levi-Civita tensor $E^{\mu \nu \alpha \beta}$.

Remarkably, the transformation (1.9) can be easily enlarged to the case of additional non-Galileon fields $\psi_{\nu_{1} \ldots}^{\mu_{1} \ldots}$. To get a minimal coupling of these fields with the Special Galileon, one simply constructs the diffeomorphism invariant Lagrangians of the nonGalileon fields on the general curved background and for the metric then substitutes the effective metric (1.9). The resulting Lagrangians are then automatically invariant under the simultaneous transformation (1.5) and the transformation of the $\psi_{\nu_{1} \ldots}^{\mu_{1} \ldots}$ fields given schematically as

$$
\delta \psi_{\nu_{1} \ldots}^{\mu_{1} \ldots}=\theta\left[-H^{\alpha \beta} \partial_{\alpha} \phi \partial_{\beta} \psi_{\nu_{1} \ldots}^{\mu_{1} \ldots}-\psi_{\alpha \ldots}^{\mu_{1} \ldots} H^{\alpha \beta} \partial_{\beta} \partial_{\nu_{1}} \phi+\psi_{\nu_{1} \ldots}^{\alpha \ldots} H^{\mu_{1} \beta} \partial_{\beta} \partial_{\alpha} \phi \ldots+\ldots\right],
$$

and therefore they can be added to the action given by the general formula (1.21). Note that the prescription (1.22) formally corresponds to the coordinate transformation

$$
\delta x^{\mu}=\theta H^{\mu \nu} \partial_{\nu} \phi
$$

For the fields which also carry spinor indices, one needs additional geometrical structures, namely the vielbain $m_{a}^{\mu}$ and the spinor connection $\beta_{b \mu}^{a}$, which are expressed in terms of the second and third derivatives of the Galileon field $\phi$ (see [21] for the explicit formulas). The invariance of the resulting action with respect to the generalized polynomial shift symmetry (1.5) then under some additional assumptions guarantees ${ }^{5}$ the enhanced $O\left(p^{3}\right)$ soft Galileon limits of the tree level scattering amplitudes.

Though the Special Galileon is well understood at the tree level, considerably less is known about its true quantum properties. In the literature, several one-loop calculations exists for the case of the cubic Galileon [27-31] and for the general Galileon [19, 32], but a systematic analysis with stress on the Special Galileon case is still missing. Namely, it is not known whether the loops do or do not break the invariance with respect to the generalized polynomial shift symmetry (1.5) of the Special Galileon, and whether the enhanced $O\left(p^{3}\right)$ soft behavior survives the quantum corrections. In this paper, we initiate the studies in this direction and try to fill this gap partially by means of the explicit calculation of the UV divergent part of the one-loop on-shell effective action using dimensional regularization (DR) and providing the complete one-loop renormalization of the $S$-matrix.

\footnotetext{
${ }^{5}$ The suffucient condition is the absence of the cubic vertices.
} 
We also calculate explicitly the lowest scattering amplitudes at one loop and discuss their compatibility with other approaches.

The paper is organized as follows. In section 2, we briefly remind the basic facts concerning the functional approach to the $S$-matrix and its relation to the on-shell effective action. Section 3 is devoted to the issue of the quantum fluctuations in the classical Galileon background, we show there that the fluctuations can be described with the action which is manifestly invariant with respect to the hidden Special Galileon symmetry. In section 4, we calculate the infinite part of the one-loop on-shell effective action for the Special Galileon and give a complete classification of the counterterms in $D=4$ for $i_{\mathrm{CT}}^{\Gamma_{1}} \leq 6$. In section 5 , we briefly discuss the extension of the soft theorem for Special Galileon to one loop. section 6 is devoted to explicit examples of the scattering amplitudes. In section 7, we summarize and further discuss our results. The technicalities concerning the classification of the counterterms are presented in appendix A.

\section{The generating functional of the $S$-matrix and the on-shell effective action}

For the reader's convenience and in order to fix our notation, let us first briefly review the interrelation of the $S$-matrix and the on-shell effective action (for original papers, see [33-35]). The starting point for our calculation is the general formula for the perturbative $S$-matrix, which we treat here as the functional $\mathcal{S}[\phi]$ of the external field $\phi$ and which is constructed $\mathrm{as}^{6}$

$$
\mathcal{S}[\phi]=\exp \left(\frac{\mathrm{i}}{\hbar} \mathcal{T}[\phi]\right)=\exp \left(-\frac{1}{2 \hbar} \frac{\delta}{\delta \phi} \cdot \mathrm{i} \Delta_{F} \cdot \frac{\delta}{\delta \phi}\right) \exp \left(\frac{\mathrm{i}}{\hbar} S_{\mathrm{int}}[\phi]\right)
$$

Here i $\Delta_{F}(x-y)$ is the Feynman propagator, $S_{\text {int }}[\phi]$ is the interaction part of the action with UV counterterms included and the functional differential operator

$$
\exp \left(-\frac{1}{2 \hbar} \frac{\delta}{\delta \phi} \cdot \mathrm{i} \Delta_{F} \cdot \frac{\delta}{\delta \phi}\right) \equiv \exp \left(-\frac{1}{2 \hbar} \int \mathrm{d}^{D} x \mathrm{~d}^{D} y \frac{\delta}{\delta \phi(x)} \mathrm{i} \Delta_{F}(x-y) \frac{\delta}{\delta \phi(y)}\right)
$$

generates formally the chronological contractions of the perturbative Wick expansion of the $S$-matrix. From the functional $\mathcal{S}[\phi]$, we can derive the operator $S$-matrix in the Dirac interaction picture inserting for $\phi$ the free field operators $\widehat{\phi}_{I}$ and treating all the operator product as normally ordered, ${ }^{7}$ namely

$$
\widehat{S}=: \mathcal{S}\left[\widehat{\phi}_{I}\right]:
$$

Therefore, it is sufficient to know $\mathcal{S}[\phi]$ on shell, i.e. for the functional argument $\phi$ satisfying the free equation of motion $\square \phi=0$.

\footnotetext{
${ }^{6}$ Here and in what follows, we often use the condensed notation where the dot means integration over the corresponding space-time coordinates, e.g.

$$
\xi \cdot O \cdot \xi \equiv \int \mathrm{d}^{D} x \mathrm{~d}^{D} y \xi(x) O(x, y) \xi(y) .
$$

${ }^{7}$ For this reason, the functional $\mathcal{S}[\phi]$ is called the normal symbol of the $S$-matrix.
} 
The connected on-shell $n$-point scattering amplitudes $A_{n}\left(p_{1}, \ldots, p_{n}\right)$ can be obtained directly from the functional $\mathcal{T}[\phi]$ by means of the functional differential operation which is equivalent to the contraction of the operators $\widehat{\phi}_{I}$ with the creation or annihilation operators in the initial or final state ${ }^{8}$

$$
A_{n}\left(p_{1}, \ldots, p_{n}\right)=\left.\int \prod_{i=1}^{n} \mathrm{~d}^{D} x_{i} \mathrm{e}^{\mathrm{i} p_{i} \cdot x_{i}} \frac{\delta}{\delta \phi\left(x_{i}\right)} \mathcal{T}[\phi]\right|_{\phi=0} .
$$

Using Gaussian integration, we can rewrite (2.1) to the form which formally includes the LSZ formulas

$$
\mathcal{S}[\phi]=\exp \left(\frac{\mathrm{i}}{\hbar} S_{0}[\phi]\right) \int D \varphi \exp \frac{\mathrm{i}}{\hbar}\left[S[\varphi]+\int \mathrm{d}^{D} x \varphi \overleftarrow{\square} \phi\right]
$$

where $S_{0}[\phi]=-\int \mathrm{d}^{D} x \phi \square \phi / 2$ is the kinetic term and where $S[\varphi]=S_{0}[\varphi]+S_{\text {int }}[\varphi]$ is the complete action with the UV counterterms included

$$
S[\varphi]=\int \mathrm{d}^{D} x\left(\mathcal{L}_{\mathrm{b}}+\mathcal{L}_{\mathrm{CT}}\right) \equiv S_{\mathrm{b}}[\varphi]+S_{\mathrm{CT}}[\varphi]=S_{0}[\varphi]+S_{\mathrm{b}, \text { int }}[\varphi]+S_{\mathrm{CT}}[\varphi]
$$

In order to obtain the loop expansion of $\mathcal{T}[\phi]$, let us substitute $\varphi=\phi_{\mathrm{cl}}+\sqrt{\hbar} \xi$, where $\phi_{\mathrm{cl}}$ is a solution of the integral equation

$$
\phi_{\mathrm{cl}}=\phi+\frac{1}{\square} \frac{\delta S_{\mathrm{b}, \text { int }}\left[\phi_{\mathrm{cl}}\right]}{\delta \phi_{\mathrm{cl}}},
$$

and $\xi$ is a new integration variable of the functional integral. Expanding now in powers of $\sqrt{\hbar}$, we get (up to an inessential overall constant)

$$
\begin{aligned}
\mathcal{S}[\phi]= & \exp \frac{\mathrm{i}}{\hbar}\left(S_{0}[\phi]+S\left[\phi_{\mathrm{cl}}\right]+\int \mathrm{d}^{D} x \phi_{\mathrm{cl}} \overleftarrow{\square} \phi\right) \\
& \times \int D \xi \exp \left(\frac{\mathrm{i}}{2} \xi \cdot \frac{\delta^{2} S_{\mathrm{b}}\left[\phi_{\mathrm{cl}}\right]}{\delta \phi_{\mathrm{cl}} \delta \phi_{\mathrm{cl}}} \cdot \xi+O\left(\hbar^{1 / 2}\right)\right) \\
= & \exp \frac{\mathrm{i}}{\hbar}\left(S_{0}[\phi]+S\left[\phi_{\mathrm{cl}}\right]+\int \mathrm{d}^{D} x \phi_{\mathrm{cl}} \overleftarrow{\square} \phi\right) \\
& \times \exp \left(\frac{\mathrm{i}}{2} \operatorname{Tr} \ln \frac{\delta^{2} S_{\mathrm{b}}\left[\phi_{\mathrm{cl}}\right]}{\delta \phi_{\mathrm{cl}} \delta \phi_{\mathrm{cl}}}\right)(1+O(\hbar)) .
\end{aligned}
$$

Let us now expand the functional $\mathcal{T}[\phi]$ and the action $S\left[\phi_{\mathrm{cl}}\right]$ in powers of $\hbar$ as

$$
\begin{aligned}
\mathcal{T}[\phi] & =\mathcal{T}^{\text {tree }}[\phi]+\hbar \mathcal{T}^{1-\text { loop }}[\phi]+O\left(\hbar^{2}\right) \\
S\left[\phi_{\mathrm{cl}}\right] & =S_{\mathrm{b}}\left[\phi_{\mathrm{cl}}\right]+\hbar S_{\mathrm{CT}}^{1-\text { loop }}\left[\phi_{\mathrm{cl}}\right]+O\left(\hbar^{2}\right),
\end{aligned}
$$

where $S_{\mathrm{CT}}^{1-\text { loop }}=\int \mathrm{d}^{D} x \mathcal{L}_{\mathrm{CT}}^{1-\text { loop }}$ are the one-loop counterterms, and $\mathcal{T}^{\text {tree }}$ and $\mathcal{T}^{1-\text { loop }}[\phi]$ are the tree and one-loop level connected $S$-matrices respectively. We can then identify

$$
\begin{aligned}
\mathcal{T}^{\text {tree }}[\phi] & =S_{0}[\phi]+S_{\mathrm{b}}\left[\phi_{\mathrm{cl}}\right]+\int \mathrm{d}^{D} x \phi_{\mathrm{cl}} \overleftarrow{\square} \phi \\
\mathcal{T}^{1-\text { loop }}[\phi] & =\frac{\mathrm{i}}{2} \operatorname{Tr} \ln \frac{\delta^{2} S_{\mathrm{b}}\left[\phi_{\mathrm{cl}}\right]}{\delta \phi_{\mathrm{cl}} \delta \phi_{\mathrm{cl}}}+S_{\mathrm{CT}}^{1-\text { loop }}\left[\phi_{\mathrm{cl}}\right] .
\end{aligned}
$$

\footnotetext{
${ }^{8}$ For simplicity, in the following formula we tacitly assume all the particles to be outgoing.
} 
Note that when understood as the functional of $\phi_{\mathrm{cl}}$, the one-loop connected $S$-matrix $\mathcal{T}^{1-\text { loop }}[\phi]$ coincides with the one-loop effective action $\Gamma^{1-\text { loop }}\left[\phi_{\mathrm{cl}}\right]$. Note also that for $\phi$ on shell (i.e. for $\square \phi=0$ ), the field $\phi_{\mathrm{cl}}$ satisfies the classical equation of motion (EOM)

$$
\square \phi_{\mathrm{cl}}=\frac{\delta S_{\mathrm{b}, \mathrm{int}}\left[\phi_{\mathrm{cl}}\right]}{\delta \phi_{\mathrm{cl}}} .
$$

Therefore, provided we are interested only in the on-shell scattering amplitudes up to one loop order, we need to know the one-loop on-shell effective action $\Gamma^{1-\text { loop }}\left[\phi_{\mathrm{cl}}\right]$, i.e. the oneloop effective action with the argument satisfying the classical equation of motion. This well known fact will be crucial for our following calculations.

\section{Quantum fluctuations in the classical background}

As discussed in the previous section, the key object for our further calculation is the operator $\delta^{2} S_{\mathrm{b}}\left[\phi_{\mathrm{cl}}\right] / \delta \phi_{\mathrm{cl}} \delta \phi_{\mathrm{cl}}$ describing the propagation of quantum fluctuations around a classical background $\phi_{\mathrm{cl}}$ which satisfies the classical equations of motion (2.13). In the case of general Galileon, the equation of motion reads (cf. (1.2))

$$
\frac{\delta S_{\mathrm{b}}\left[\phi_{\mathrm{cl}}\right]}{\delta \phi_{\mathrm{cl}}}=\sum_{n=0}^{D}(n+1) d_{n+1} \varepsilon^{\mu_{1} \ldots \mu_{D}} \varepsilon^{\nu_{1} \ldots \nu_{D}} \prod_{i=1}^{n} \partial_{\mu_{i}} \partial_{\nu_{i}} \phi_{\mathrm{cl}} \prod_{j=n+1}^{D} \eta_{\mu_{j} \nu_{j}}=0
$$

Taking the second functional derivative, we derive for the fluctuation operator

$$
\begin{aligned}
\frac{\delta^{2} S_{\mathrm{b}}\left[\phi_{\mathrm{cl}}\right]}{\delta \phi_{\mathrm{cl}}(x) \delta \phi_{\mathrm{cl}}(y)}= & \sum_{n=1}^{D}(n+1) n d_{n+1} \varepsilon^{\mu_{1} \ldots \mu_{D}} \varepsilon^{\nu_{1} \ldots \nu_{D}} \prod_{i=1}^{n-1} \partial_{\mu_{i}} \partial_{\nu_{i}} \phi_{\mathrm{cl}}(x) \\
& \times \prod_{j=n+1}^{D} \eta_{\mu_{j} \nu_{j}} \partial_{\mu_{n}} \partial_{\nu_{n}} \delta^{(D)}(x-y) \\
= & -G^{\mu \nu}\left[\phi_{\mathrm{cl}}\right] \partial_{\mu} \partial_{\nu} \delta^{(D)}(x-y),
\end{aligned}
$$

where we have denoted

$$
\begin{aligned}
G^{\mu \nu}\left[\phi_{\mathrm{cl}}\right] & =-\frac{\partial}{\partial\left[\partial_{\mu} \partial_{\nu} \phi_{\mathrm{cl}}\right]} \frac{\delta S_{\mathrm{b}}\left[\phi_{\mathrm{cl}}\right]}{\delta \phi_{\mathrm{cl}}} \\
& =\sum_{n=1}^{D}(n+1) n d_{n+1} \varepsilon^{\mu_{1} \ldots \mu_{D}} \varepsilon^{\nu_{1} \ldots \nu_{D}} \prod_{i=1}^{n-1} \partial_{\mu_{i}} \partial_{\nu_{i}} \phi_{\mathrm{cl}}(x) \prod_{j=n+1}^{D} \eta_{\mu_{j} \nu_{j}} .
\end{aligned}
$$

Note that $\partial_{\mu} G^{\mu \nu}\left[\phi_{\mathrm{cl}}\right]=\partial_{\nu} G^{\mu \nu}\left[\phi_{\mathrm{cl}}\right]=0$, and therefore the quantum fluctuations in the quadratic approximation are described by the action

$$
\begin{aligned}
S_{q}\left[\phi_{\mathrm{cl}}, \xi\right] & =\frac{1}{2} \xi \cdot \frac{\delta^{2} S_{\mathrm{b}}\left[\phi_{\mathrm{cl}}\right]}{\delta \phi_{\mathrm{cl}} \delta \phi_{\mathrm{cl}}} \cdot \xi \\
& =\frac{1}{2} \int \mathrm{d}^{D} x G^{\mu \nu}\left[\phi_{\mathrm{cl}}\right](x) \partial_{\mu} \xi(x) \partial_{\nu} \xi(x),
\end{aligned}
$$


In the case of the Special Galileon, we can sum up the right hand side of (3.3) and (3.4) in a closed form. Indeed, inserting (1.3) with $\beta=0$, we get for the first branch of the Galileon Lagrangians ${ }^{9}$

$$
\begin{aligned}
\frac{\delta S_{\mathrm{b}}^{(+)}\left[\phi_{\mathrm{cl}}\right]}{\delta \phi_{\mathrm{cl}}} & =-\frac{\mathrm{i} \alpha(-1)^{D}}{D !} \sum_{n=1}^{\left[\frac{D}{2}\right]}\left(\begin{array}{c}
D \\
2 n-1
\end{array}\right)\left(\frac{\mathrm{i}}{\alpha}\right)^{2 n-1} \varepsilon^{\mu_{1} \ldots \mu_{D}} \varepsilon^{\nu_{1} \ldots \nu_{D}} \prod_{i=1}^{2 n-1} \partial_{\mu_{i}} \partial_{\nu_{i}} \phi_{\mathrm{cl}} \prod_{j=2 n-1}^{D} \eta_{\mu_{j} \nu_{j}} \\
& =-\frac{\mathrm{i} \alpha(-1)^{D}}{2 D !} \varepsilon^{\mu_{1} \ldots \mu_{D}} \varepsilon^{\nu_{1} \ldots \nu_{D}}\left[\prod_{i=1}^{D}\left(\eta_{\mu_{i} \nu_{i}}+\frac{\mathrm{i}}{\alpha} \partial_{\mu_{i}} \partial_{\nu_{i}} \phi_{\mathrm{cl}}\right)-\prod_{i=1}^{D}\left(\eta_{\mu_{i} \nu_{i}}-\frac{\mathrm{i}}{\alpha} \partial_{\mu_{i}} \partial_{\nu_{i}} \phi_{\mathrm{cl}}\right)\right] .
\end{aligned}
$$

Using the general identity valid for any $D \times D$ matrix $M_{\mu \nu}$

$$
\varepsilon^{\mu_{1} \ldots \mu_{D}} \varepsilon^{\nu_{1} \ldots \nu_{D}} \prod_{i=1}^{D} M_{\mu_{i} \nu_{i}}=D ! \operatorname{det} M_{\mu \nu}=(-1)^{D-1} D ! \operatorname{det} M_{\nu}^{\mu},
$$

we can rewrite the EOM in a compact form as

$$
\frac{\delta S_{\mathrm{b}}^{(+)}\left[\phi_{\mathrm{cl}}\right]}{\delta \phi_{\mathrm{cl}}}=\frac{\mathrm{i} \alpha}{2}\left(\mathcal{D}_{+}^{(+)}-\mathcal{D}_{-}^{(+)}\right)=0
$$

where we have denoted

$$
\mathcal{D}_{ \pm}^{(+)}=\operatorname{det}\left(\delta_{\nu}^{\mu} \pm \frac{\mathrm{i}}{\alpha} \partial^{\mu} \partial_{\nu} \phi_{\mathrm{cl}}\right) .
$$

In the same way, using instead (1.4) for the second branch of the Special Galileon Lagrangians, we get

$$
\frac{\delta S_{\mathrm{b}}^{(-)}\left[\phi_{\mathrm{cl}}\right]}{\delta \phi_{\mathrm{cl}}}=-\frac{\alpha}{2}\left(\mathcal{D}_{+}^{(-)}-\mathcal{D}_{-}^{(-)}\right)=0
$$

where now

$$
\mathcal{D}_{ \pm}^{(-)}=\operatorname{det}\left(\delta_{\nu}^{\mu} \pm \frac{1}{\alpha} \partial^{\mu} \partial_{\nu} \phi_{\mathrm{cl}}\right)
$$

Taking the partial derivative of (3.7) and (3.9) with respect to $\partial_{\mu} \partial_{\nu} \phi_{\mathrm{cl}}$, we get

$$
\begin{aligned}
& G_{(+)}^{\mu \nu}\left[\phi_{\mathrm{cl}}\right]=\frac{1}{2} \eta^{\mu \alpha}\left[\mathcal{D}_{+}^{(+)}\left(\mathbf{1}+\frac{\mathrm{i}}{\alpha} \boldsymbol{\partial} \boldsymbol{\partial} \phi_{\mathrm{cl}}\right)^{-1}+\mathcal{D}_{-}^{(+)}\left(\mathbf{1}-\frac{\mathrm{i}}{\alpha} \boldsymbol{\partial} \boldsymbol{\partial} \phi_{\mathrm{cl}}\right)^{-1}\right]_{\alpha}^{\nu} \\
& G_{(-)}^{\mu \nu}\left[\phi_{\mathrm{cl}}\right]=\frac{1}{2} \eta^{\mu \alpha}\left[\mathcal{D}_{+}^{(-)}\left(\mathbf{1}+\frac{1}{\alpha} \boldsymbol{\partial} \boldsymbol{\partial} \phi_{\mathrm{cl}}\right)^{-1}+\mathcal{D}_{-}^{(-)}\left(\mathbf{1}-\frac{1}{\alpha} \boldsymbol{\partial} \boldsymbol{\partial} \phi_{\mathrm{cl}}\right)^{-1}\right]_{\alpha}^{\nu}
\end{aligned}
$$

where $\boldsymbol{\partial} \boldsymbol{\partial} \phi_{\mathrm{cl}}$ denotes the $D \times D$ matrix with elements $\partial^{\mu} \partial_{\nu} \phi_{\mathrm{cl}}$. This can be further simplified using the EOM (3.7) and (3.9) in the form ${ }^{10}$

$$
\mathcal{D}_{+}^{(+)}=\mathcal{D}_{-}^{(+)}=\sqrt{\mathcal{D}_{+}^{(+)} \mathcal{D}_{-}^{(+)}},
$$

\footnotetext{
${ }^{9}$ We have rescaled the coefficients $d_{n}^{( \pm)}$by a factor $\pm(-1)^{D} / D$ ! in comparisson with (1.3) and (1.4) in order to ensure the canonical normalization of the kinetic term.

${ }^{10}$ Note that EOM ensures that $\mathcal{D}_{+}^{(+)}=\mathcal{D}_{-}^{(+)}$is real. Here we further suppose that $\mathcal{D}_{+}^{(+)}$is positive as the weak field limit $\mathcal{D}_{+}^{(+)}=1+O\left(\partial \partial \phi_{\mathrm{cl}}\right)$ suggests.
} 
with the result

$$
\begin{aligned}
G_{(+)}^{\mu \nu}\left[\phi_{\mathrm{cl}}\right] & =\eta^{\mu \alpha} \sqrt{\mathcal{D}_{+}^{(+)} \mathcal{D}_{-}^{(+)}}\left[\left(1+\frac{1}{\alpha^{2}} \boldsymbol{\partial} \boldsymbol{\partial} \phi_{\mathrm{cl}} \cdot \boldsymbol{\partial} \boldsymbol{\partial} \phi_{\mathrm{cl}}\right)^{-1}\right]_{\alpha}^{\nu} \\
& =\sqrt{g} g^{\mu \nu}
\end{aligned}
$$

and similarly $G_{(-)}^{\mu \nu}\left[\phi_{\mathrm{cl}}\right]=\sqrt{g} g^{\mu \nu}$. Here $g^{\mu \nu}$ is the matrix inverse of the Special Galileon effective metric $g_{\mu \nu}=\eta_{\mu \nu} \pm \partial_{\mu} \partial \phi_{\mathrm{cl}} \cdot \partial \partial_{\nu} \phi_{\mathrm{cl}} / \alpha^{2}$ (see (1.9)) and $g=\left|\operatorname{det} g_{\mu \nu}\right|$ is its determinant. The fluctuation action (3.5) is thus rewritten in the geometrical form ${ }^{11}$

$$
S_{q}\left[\phi_{\mathrm{cl}}, \xi\right]=\frac{1}{2} \int \mathrm{d}^{D} x \sqrt{g} g^{\mu \nu} \partial_{\mu} \xi \partial_{\nu} \xi
$$

Therefore, it is invariant under the hidden Special Galileon transformation (1.5) of the Galileon field $\phi_{\mathrm{cl}}$ and a simultaneous transformation of the field $\xi$ according to (cf. (1.22))

$$
\delta \xi=-\theta H^{\alpha \beta} \partial_{\alpha} \phi_{\mathrm{cl}} \partial_{\beta} \xi
$$

This can be used for a formal proof of the invariance of the pure loop part of the on-shell effective action $\Gamma_{\text {loop }}^{1-\text { loop }}\left[\phi_{\mathrm{cl}}\right]$ with respect to the hidden Special Galileon symmetry. Indeed, up to an inessential constant, ${ }^{12}$

$$
\Gamma_{\text {loop }}^{1-\text { loop }}\left[\phi_{\mathrm{cl}}\right]=-\mathrm{i} \ln \int D \xi \exp \left(\mathrm{i} S_{q}\left[\phi_{\mathrm{cl}}, \xi\right]\right)
$$

where an appropriate regularization compatible with the symmetry (e.g. dimensional regularization will do the job as we will discussed later) is tacitly assumed. Thus under the transformations (1.5) and (3.16)

$$
\begin{aligned}
\Gamma_{\text {loop }}^{1-\text { loop }}\left[\phi_{\mathrm{cl}}+\delta \phi_{\mathrm{cl}}\right] & =-\mathrm{i} \ln \int D(\xi+\delta \xi) \exp \left(\mathrm{i} S_{q}\left[\phi_{\mathrm{cl}}+\delta \phi_{\mathrm{cl}}, \xi+\delta \xi\right]\right) \\
& =-\mathrm{i} \ln \int D \xi J \exp \left(\mathrm{i} S_{q}\left[\phi_{\mathrm{cl}}, \xi\right]\right),
\end{aligned}
$$

where the Jacobian $J=1-\theta \operatorname{Tr}\left(H^{\alpha \beta} \partial_{\alpha} \phi_{\mathrm{cl}} \partial_{\beta}\right)$ can be shown to be equal to one (this holds within dimensional regularization — see e.g. a similar calculation in [19]), which proves the statement.

\footnotetext{
${ }^{11}$ Let us note that in the case of general Galileon in $D \neq 2$ dimensions, the fluctuation action (3.5) can be also rewritten in the geometric form

$$
S_{q}\left[\phi_{\mathrm{cl}}, \xi\right]=\frac{1}{2} \int \mathrm{d}^{D} x \sqrt{\mathcal{G}} \mathcal{G}^{\mu \nu} \partial_{\mu} \xi \partial_{\nu} \xi
$$

where now

$$
\mathcal{G}^{\mu \nu}=\left|\operatorname{det} G^{\mu \nu}\left[\phi_{\mathrm{cl}}\right]\right|^{\frac{1}{2-D}} G^{\mu \nu}\left[\phi_{\mathrm{cl}}\right] .
$$

The exclusivity of the Special Galileons lies in the fact that in this case the inverse metric $G_{\mu \nu}$ is computable and equals to the explicitly known effective metric $g_{\mu \nu}$. This ensures the invariance of the fluctuation action with respect to the hidden Special Galileon symmetry.

${ }^{12}$ From now on, we return to the natural units $\hbar=1$.
} 
In what follows, we will concentrate on the UV divergent part of $\Gamma_{\text {loop }}^{1-\text { loop }}\left[\phi_{\mathrm{cl}}\right]_{\infty}$, which is local, and which determines the UV divergent part of the one-loop counterterm action $S_{\mathrm{CT}}^{1-\text { loop }}\left[\phi_{\mathrm{cl}}\right]$

$$
S_{\mathrm{CT}}^{1-\mathrm{loop}}\left[\phi_{\mathrm{cl}}\right]=-\Gamma_{\text {loop }}^{1-\mathrm{loop}}\left[\phi_{\mathrm{cl}}\right]_{\infty}+S_{\mathrm{CT}}^{1-\mathrm{loop}}\left[\phi_{\mathrm{cl}}\right]_{\mathrm{finite}},
$$

where $S_{\mathrm{CT}}^{1-\text { loop }}\left[\phi_{\mathrm{cl}}\right]_{\text {finite }}$ includes all the finite parts of the couterterms. According to (3.18), the divergent part of $S_{\mathrm{CT}}^{1-\text { loop }}\left[\phi_{\mathrm{cl}}\right]$ is invariant with respect to the hidden Special Galileon symmetry. In the next section, we prove this statement less formally by the explicit calculation of $\Gamma_{\text {loop }}^{1-\text { loop }}\left[\phi_{\mathrm{cl}}\right]_{\infty}$ within dimensional regularization.

\section{The UV divergences at one loop}

The most economic way how to obtain the UV divergent part of $\Gamma_{\text {loop }}^{1-\text { loop }}\left[\phi_{\mathrm{cl}}\right]$ within dimensional regularization is to use the master formula stemming from the heat kernel expansion [36, 37]. From (3.15), it follows that

$$
S_{q}\left[\phi_{\mathrm{cl}}, \xi\right]=-\frac{1}{2} \int \mathrm{d}^{D} x \sqrt{g} \xi g^{\mu \nu} D_{\mu} D_{\nu} \xi
$$

where $D_{\mu}$ is the covariant derivative associated with the effective metric $g_{\mu \nu}$. Therefore, up to an inessential constant, we can express $\Gamma_{\text {loop }}^{1-\text { loop }}\left[\phi_{\mathrm{cl}}\right]$ in terms of the heat kernel (here we use the Lorentzian signature, see e.g. [38])

$$
K(\tau ; x, y)=\exp \left[\mathrm{i} \tau\left(g^{\mu \nu} D_{\mu} D_{\nu}+\mathrm{i} 0\right)\right]
$$

using the well known formula for logarithm of the operator determinant

$$
\Gamma_{\text {loop }}^{1-\text { loop }}\left[\phi_{\mathrm{cl}}\right]=\frac{\mathrm{i}}{2} \int \mathrm{d}^{D} x \sqrt{g} \int_{0}^{\infty} \frac{\mathrm{d} \tau}{\tau} K(\tau ; x, x) .
$$

At the coincident points $x=y$ the heat kernel $K(\tau ; x, y)$ has the following asymptotic expansion for $\tau \rightarrow 0$

$$
K(\tau ; x, x)=\frac{\mathrm{i}}{(4 \pi \mathrm{i} \tau)^{D / 2}} \sum_{n=0}^{\infty}(\mathrm{i} \tau)^{n} a_{n}(x) \mathrm{e}^{-\tau 0},
$$

where $a_{n}(x)$ 's are the coincidence limits of the Seeley-DeWitt coefficients. The UV divergences of the effective action $\Gamma_{\text {loop }}^{1-\text { loop }}\left[\phi_{\mathrm{cl}}\right]$ are then connected with the lower limit of the integral in the formula (4.3) and can be cured by dimensional regularization writing $D \rightarrow D-2 \varepsilon$. This gives the master formula valid for $D$ even ${ }^{13}$

$$
\Gamma_{\text {loop }}^{1-\text { loop }}\left[\phi_{\mathrm{cl}}\right]_{\infty}=\frac{\mu^{-2 \varepsilon}}{2(4 \pi)^{D / 2}} \frac{1}{\varepsilon} \int \mathrm{d}^{D} x \sqrt{g} a_{D / 2}(x),
$$

\footnotetext{
${ }^{13}$ For $D$ odd, all the one loop infinities are removed automatically by means of the dimensional continuation.
} 
where $\mu$ is the dimensional renormalization scale parameter. The Seeley-DeWitt coefficients $a_{n}(x)$ are calculable and explicitly known for $n \leq 10$ in terms of the geometrical invariants (for a review and a complete list of references see [39]), e.g.

$$
\begin{aligned}
& a_{0}=1 \\
& a_{1}=\frac{1}{6} R \\
& a_{2}=\frac{1}{180}\left(R_{\alpha \beta \mu \nu} R^{\alpha \beta \mu \nu}-R_{\mu \nu} R^{\mu \nu}\right)+\frac{1}{72} R^{2}+\frac{1}{30} g^{\mu \nu} D_{\mu} D_{\nu} R \\
& a_{3}=\frac{1}{7 !}\left(18 g^{\mu \nu} g^{\alpha \beta} D_{\mu} D_{\nu} D_{\alpha} D_{\beta} R+17 g^{\mu \nu} D_{\mu} R D_{\nu} R+\ldots\right) .
\end{aligned}
$$

Here $R_{\alpha \beta \mu \nu}, R_{\mu \nu}$, and $R$ are the Riemann curvature tensor, the Ricci tensor, and the scalar curvature respectively corresponding in our case to the effective metric $g_{\mu \nu}$. All these objects can be expressed in terms of the extrinsic curvature tensor (1.10) and the inverse metric $g^{\mu \nu}$, namely (see [21] for more details)

$$
R_{\alpha \beta \mu \nu}=g^{\rho \sigma}\left(\mathcal{K}_{\rho \mu \alpha} \mathcal{K}_{\sigma \nu \beta}-\mathcal{K}_{\rho \mu \beta} \mathcal{K}_{\sigma \nu \alpha}\right) .
$$

As discussed in the introduction, due to their geometrical nature, all the Seeley-DeWitt coefficients are then automatically invariant with respect to the hidden Special Galileon symmetry and so is the divergent part of the one-loop on-shell effective action (4.5).

In what follows, we will concentrate on the four-dimensional case. Note that we can further simplify the above formulas dropping the last term in $a_{2}(x)$ which is a total derivative and also eliminating a total derivative corresponding to the four-dimensional GaussBonett term

$$
G=R_{\alpha \beta \mu \nu} R^{\alpha \beta \mu \nu}-4 R_{\mu \nu} R^{\mu \nu}+R^{2}
$$

The result then reads

$$
\Gamma_{\text {loop }}^{1-\text { loop }}\left[\phi_{\mathrm{cl}}\right]_{\infty}^{D=4}=\frac{\mu^{-2 \varepsilon}}{(4 \pi)^{2}} \frac{1}{120} \frac{1}{\varepsilon} \int \mathrm{d}^{4} x \sqrt{g}\left(R_{\mu \nu} R^{\mu \nu}+\frac{1}{2} R^{2}\right) .
$$

Note that the index of the vertices of (4.9) equals to (note that $g_{\mu \nu}$ contains exactly two derivatives per field, while $\mathcal{K}_{\sigma \nu \beta}$ has three derivatives per field, and that $R R \sim \mathcal{K}^{4}$ )

$$
i_{V}=\left(2 E_{V}+4\right)-\left(2 E_{V}-2\right)=6 .
$$

This coincides with the index of the corresponding one-loop graphs (cf (1.8)). Dimensional regularization therefore respects the hierarchy of the counterterms, especially the basic Lagrangian is not renormalized. This is not true for other regularization schemes, e.g. for the momentum cutoff corresponding to the deformation of the integral in (4.3) by means of introducing lower limit of the integration of the order $1 / \Lambda^{2}$. In such a case, all the SeeleyDeWitt coefficients up to $n=2\left(\right.$ or $n=D / 2$ for general $D$ ) contribute to $\Gamma_{\text {loop }}^{1-\text { loop }}\left[\phi_{\mathrm{cl}}\right]_{\infty}$.

Because we are concentrated on the $S$-matrix and on-shell amplitudes, we can simplify (4.9) further using the classical EOM. Indeed, taking a derivative of (3.1), we get

$$
\sum_{n=0}^{D}(n+1) n d_{n+1} \varepsilon^{\mu_{1} \ldots \mu_{D}} \varepsilon^{\nu_{1} \ldots \nu_{D}} \prod_{i=1}^{n-1} \partial_{\mu_{i}} \partial_{\nu_{i}} \phi_{\mathrm{cl}} \prod_{j=n+1}^{D} \eta_{\mu_{j} \nu_{j}} \partial_{\alpha} \partial_{\mu_{n}} \partial_{\nu_{n}} \phi_{\mathrm{cl}}=0
$$


which can be rewritten with help of (3.4), (3.14) and (1.10) as

$$
-\alpha \sqrt{g} g^{\mu \nu} \mathcal{K}_{\mu \nu \alpha}=0
$$

The Ricci tensor given by eq. (4.7) then simplifies

$$
R_{\mu \nu}=-g^{\alpha \beta} g^{\rho \sigma} \mathcal{K}_{\rho \beta \mu} \mathcal{K}_{\sigma \nu \alpha} \equiv-\frac{1}{\alpha^{2}} \partial_{\mu} \partial \partial \phi_{\mathrm{cl}}: \partial \partial \partial_{\nu} \phi_{\mathrm{cl}}
$$

where the bold colon (and in general the bold dot in what follows) denotes the contraction of the adjacent indices with the help of the inverse effective metric $g^{\kappa \lambda}$. Using the same shorthand notation, we get for the scalar curvature

$$
R=-\langle\langle\mathcal{K}: \mathcal{K}\rangle\rangle=-\frac{1}{\alpha^{2}}\left\langle\left\langle\partial \partial \partial \phi_{\mathrm{cl}}: \partial \partial \partial \phi_{\mathrm{cl}}\right\rangle\right\rangle
$$

where $\langle\langle\cdot\rangle\rangle$ denotes the trace with respect to the inverse metric $g^{\kappa \lambda}$. Finally, we get

$$
\left.\Gamma_{\text {loop }}^{1-\text { loop }}\left[\phi_{\text {cl }}\right]_{\infty}^{D=4}=\frac{\mu^{-2 \varepsilon}}{(4 \pi)^{2}} \frac{1}{120} \frac{1}{\varepsilon} \int \mathrm{d}^{4} x \sqrt{g}[\langle\mathcal{K}: \mathcal{K} \cdot \mathcal{K}: \mathcal{K}\rangle\rangle+\frac{1}{2}\langle\langle\mathcal{K}: \mathcal{K}\rangle\rangle^{2}\right]
$$

or more explicitly

$$
\begin{aligned}
\Gamma_{\text {loop }}^{1-\text { loop }}\left[\phi_{\mathrm{cl}}\right]_{\infty}^{D=4}= & \frac{\mu^{-2 \varepsilon}}{(4 \pi)^{2}} \frac{1}{120 \alpha^{4}} \frac{1}{\varepsilon} \int \mathrm{d}^{4} x \sqrt{\left|\operatorname{det}\left(\eta \pm \frac{1}{\alpha^{2}} \partial \partial \phi_{\mathrm{cl}} \cdot \partial \partial \phi_{\mathrm{cl}}\right)\right|} \\
& \times\left[\left\langle\left\langle\partial \partial \partial \phi_{\mathrm{cl}}: \partial \partial \partial \phi_{\mathrm{cl}} \cdot \partial \partial \partial \phi_{\mathrm{cl}}: \partial \partial \partial \phi_{\mathrm{cl}}\right\rangle\right\rangle\right. \\
& \left.+\frac{1}{2}\left\langle\left\langle\partial \partial \partial \phi_{\mathrm{cl}}: \partial \partial \partial \phi_{\mathrm{cl}}\right\rangle\right\rangle^{2}\right]
\end{aligned}
$$

The two operators on the right hand sides of the last two equations are therefore the only operators invariant with respect to the hidden Special Galileon symmetry which have to be inserted with infinite coefficients into the counterterm action $S_{\mathrm{CT}}^{1-\text { loop }}$. Interestingly, there are no other non-vanishing invariant operators of the form $\mathcal{K}^{4}$ provided the EOM constraint (4.11) is satisfied. ${ }^{14}$

The one-loop counterterm action which is necessary to renormalize the infinities of the on-shell amplitudes up to and including the graphs with the graph index $i_{\mathrm{CT}}^{\Gamma_{\mathrm{T}}} \leq 6$ (cf. (1.8)) therefore reads

$$
S_{\mathrm{CT}}^{1-\text { loop }}=\frac{\mu^{-2 \varepsilon}}{(4 \pi)^{2}} \frac{1}{120} \frac{\alpha^{2}}{\Lambda^{6}} \int \mathrm{d}^{4} x \sqrt{g}\left[c_{1} \mathcal{O}_{1}+c_{2} \mathcal{O}_{2}\right]+S_{\mathrm{CT}, \text { finite }}^{1-\text { loop }}
$$

where we have denoted

$$
\begin{aligned}
& \mathcal{O}_{1}=R_{\mu \nu} R^{\mu \nu}=\langle\langle\mathcal{K}: \mathcal{K} \cdot \mathcal{K}: \mathcal{K}\rangle\rangle \\
& \mathcal{O}_{2}=R^{2}=\langle\langle\mathcal{K}: \mathcal{K}\rangle\rangle^{2} .
\end{aligned}
$$

\footnotetext{
${ }^{14}$ See appendix A for more details.
} 
In the formula (4.16), $\Lambda$ is the scale which controls the systematic derivative expansion, or more precisely, the expansion in the graph index $i_{\mathrm{CT}}^{\Gamma}$, and $c_{i}$ are dimensionless bare couplings to be specified later. The finite part of the counterterm action $S_{\mathrm{CT}}^{1-\text { loop }}$ finite has the general form (1.21) . However, provided the EOM (3.7) or (3.9) are satisfied, the invariant $\sigma$ and their covariant derivatives do not correspond to the independent building blocks. Indeed, using the EOM in the form (3.7), we get from (1.18) for the "plus" branch

$$
\sigma=\frac{\alpha}{2 \mathrm{i}} \ln \left[\frac{\mathcal{D}_{+}^{(+)}}{\mathcal{D}_{-}^{(+)}}\right]=0
$$

and analogicaly for the "minus" branch. As a result, $S_{\mathrm{CT}}^{1-\text { loop }}$ finite can be written without loss of generality in the form

$$
S_{\mathrm{CT}, \text { finite }}^{1-\text { loop }}=\mu^{-2 \varepsilon} \int \mathrm{d}^{4} x \sqrt{g} \sum_{j>2} \frac{\alpha^{2}}{\Lambda^{i_{\mathcal{O}_{j}}}} c_{j} \mathcal{O}_{j},
$$

where the operators $\mathcal{O}_{j}, j>2$ are given schematically as $\mathcal{O}_{j}=D^{n_{j}} \mathcal{K}^{m_{j}}$. The index of the corresponding terms in the action is then simply ${ }^{15}$

$$
i_{\mathcal{O}_{j}}=n_{j}+m_{j}+2 .
$$

The operators $\mathcal{O}_{j}, j>2$, together with $\mathcal{O}_{1}$ and $\mathcal{O}_{2}$ should form a complete ${ }^{16}$ set of operators with index $i_{\mathcal{O}_{j}} \leq 6$, which are invariant with respect to the hidden Special Galileon symmetry. There are only two operators with $i_{\mathcal{O}_{j}}<6$ which do not vanish as a consequence of $\operatorname{EOM}(4.11)$, namely ${ }^{17}$

$$
\begin{aligned}
\mathcal{O}_{3} & =\langle\langle\mathcal{K}: \mathcal{K}\rangle\rangle=-R, \\
\mathcal{O}_{4} & =1,
\end{aligned}
$$

with $i_{\mathcal{O}_{3}}=4$ and $i_{\mathcal{O}_{4}}=2$ respectively (cf. [21] for further discussion). For $i_{\mathcal{O}_{j}}=6$, there is on top of $\mathcal{O}_{1}$ and $\mathcal{O}_{2}$ only one (up to integration by parts) invariant operator with one covariant derivative

$$
\mathcal{O}_{5}=\langle\langle(\mathcal{K}: \mathcal{K}) \cdot(D \cdot \mathcal{K})\rangle\rangle,
$$

and we get two invariant operators with two covariant derivatives

$$
\begin{aligned}
& \mathcal{O}_{6}=(D \cdot \mathcal{K}):(D \cdot \mathcal{K}) \\
& \mathcal{O}_{7}=\langle\langle(\mathcal{K}:(D \cdot D) \mathcal{K})\rangle\rangle .
\end{aligned}
$$

However, as shown in the appendix $\mathrm{A}$, the operators $\mathcal{O}_{5}$ and $\mathcal{O}_{6}$ vanish due to the relation

$$
D \cdot \mathcal{K}_{\mu \nu}=g^{\alpha \beta} D_{\alpha} \mathcal{K}_{\beta \mu \nu}=0,
$$

\footnotetext{
${ }^{15}$ Note that the measure $\mathrm{d}^{4} x \sqrt{g}$ does not contribute to the index since it contains two derivatives per field.

${ }^{16}$ Here complete means modulo integration by parts and use of EOM.

${ }^{17}$ These two operators correspond to the quartic and quadratic divergences respectively when the momentum cutoff is used instead of dimensional regularization.
} 
which is valid as a consequence of EOM, and the operator $\mathcal{O}_{7}$ can be rewritten (again using EOM and integration by parts) as a linear combination of the operators $\mathcal{O}_{1}$ and $\mathcal{O}_{2}$ plus a total derivative. The same is true for the apparently independent operator

$$
\mathcal{O}_{8}=g^{\kappa \rho} g^{\gamma \beta} g^{\delta \alpha} g^{\mu \nu} \mathcal{K}_{\kappa \gamma \delta} \mathcal{K}_{\nu \rho} \cdot \mathcal{K}_{\beta} \cdot \mathcal{K}_{\alpha \mu}
$$

The parity odd operators with $i_{\mathcal{O}_{j}} \leq 6$ of the type

$$
\mathcal{O}_{j}=\left(D^{n_{j}} \mathcal{K}^{m_{j}}\right)_{\mu_{1} \mu_{2} \mu_{3} \mu_{4}} E^{\mu_{1} \mu_{2} \mu_{3} \mu_{4}}
$$

where $E^{\mu_{1} \mu_{2} \mu_{3} \mu_{4}}$ is the contravariant Levi-Civita tensor (1.12) vanish on-shell due to the symmetry properties of the building blocks (see appendix A).

To summarize, there are only four independent on-shell counterterms, interestingly just those which already appeared in the Seeley-DeWitt coefficients (4.6)

$$
\begin{aligned}
S_{\mathrm{CT}}^{1-\text { loop }} & =\mu^{-2 \varepsilon} \int \mathrm{d}^{4} x \sqrt{g} \sum_{j=1}^{4} \frac{\alpha^{2}}{\Lambda^{i \mathcal{O}_{j}}} c_{j} \mathcal{O}_{j} \\
& =\mu^{-2 \varepsilon} \frac{\alpha^{2}}{\Lambda^{2}} \int \mathrm{d}^{4} x \sqrt{g}\left(\frac{c_{1}}{\Lambda^{4}} R_{\mu \nu} R^{\mu \nu}+\frac{c_{2}}{\Lambda^{4}} R^{2}+\frac{c_{3}}{\Lambda^{2}} R+c_{4}\right) .
\end{aligned}
$$

The bare parameters $c_{i}$ are expressed in terms of the finite couplings $c_{i}^{\mathrm{r}}(\mu)$ renormalized at the scale $\mu$ according to ${ }^{18}$

$$
\begin{aligned}
c_{i} & =c_{i}^{\mathrm{r}}(\mu)+\frac{\beta_{i}}{\varepsilon}, \\
\beta_{1} & =-\frac{\Lambda^{6}}{120(4 \pi \alpha)^{2}}, \quad \beta_{2}=-\frac{\Lambda^{6}}{240(4 \pi \alpha)^{2}}, \quad \beta_{i>2}=0
\end{aligned}
$$

and their renormalization scale dependence is determined in terms of the coefficients $\beta_{i}$ as

$$
c_{i}^{\mathrm{r}}\left(\mu^{\prime}\right)=c_{i}^{\mathrm{r}}(\mu)+2 \beta_{i} \ln \left(\frac{\mu^{\prime}}{\mu}\right) .
$$

The natural values of $c_{i}^{\mathrm{r}}(\mu)$ is of the order $O(1)$. Therefore, in order to avoid incommensurable effects stemming from the counterterms and from the loops, we expect $2 \beta_{j}=O(1)$ and thus the natural value of the scale $\Lambda$ is

$$
\Lambda \simeq 2(4 \pi \alpha)^{1 / 3} \simeq 4.6 \times \alpha^{1 / 3}
$$

Thus the consistency of the loop expansion requires that the scale $\alpha^{1 / 3}$ which controls the strength of non-linearities in the basic Lagrangian is roughly of the same order as the scale $\Lambda$ which controls the systematic expansion of the quantum corrections.

\footnotetext{
${ }^{18}$ Here we use the renormalization scheme suitable for power counting non-renormalizable theories described in detail in [40].
} 


\section{The scattering amplitudes at one loop and the soft theorem}

Using the results of the previous section, we can write the renormalized (i.e. finite) one-loop $S$-matrix in the form

$$
\mathcal{T}^{\text {tree }}[\phi]+\mathcal{T}^{1-\text { loop }}[\phi]=S_{0}[\phi]+S_{\text {eff }}^{1-\text { loop }}\left[\phi_{\mathrm{cl}}\right]+\int \mathrm{d}^{D} x \phi_{\mathrm{cl}} \overleftarrow{\square} \phi
$$

where we have denoted as $S_{\text {eff }}^{1-\text { loop }}\left[\phi_{\mathrm{cl}}\right]$ the non-local one-loop effective action given by

$$
S_{\text {eff }}^{1-\text { loop }}\left[\phi_{\mathrm{cl}}\right]=S_{\mathrm{b}}\left[\phi_{\mathrm{cl}}\right]+S_{\mathrm{CT}}^{1-\text { loop }}\left[\phi_{\mathrm{cl}}\right]+\Gamma_{\text {loop }}^{1-\text { loop }}\left[\phi_{\mathrm{cl}}\right]
$$

with

$$
\Gamma_{\text {loop }}^{1-\text { loop }}\left[\phi_{\mathrm{cl}}\right]=\frac{\mathrm{i}}{2} \operatorname{Tr} \ln \frac{\delta^{2} S_{\mathrm{b}}\left[\phi_{\mathrm{cl}}\right]}{\delta \phi_{\mathrm{cl}} \delta \phi_{\mathrm{cl}}} .
$$

Note that $\phi_{\mathrm{cl}}$ is still determined by (2.7). Let us now change for a moment the definition of $\phi_{\mathrm{cl}}$ according to

$$
\phi_{\mathrm{cl}}=\phi+\frac{1}{\square} \frac{\delta S_{\mathrm{eff}}^{1-\mathrm{loop}, \mathrm{int}}\left[\phi_{\mathrm{cl}}\right]}{\delta \phi_{\mathrm{cl}}},
$$

where $S_{\text {eff }}^{1-\text { loop, int }}$ is the interaction part of the effective one-loop action $S_{\text {eff }}^{1-\text { loop }}$. Then we can compare (5.1) and (5.4) with the tree-level formulas (2.11) and (2.7). Note that the latter correspond to amplitudes given as a sum of the tree graphs built from free propagators and vertices derived from the basic interaction action $S_{\mathrm{b}}^{\text {int }}$. Therefore, we can conclude that the modified prescription (5.1) and (5.4) corresponds to the scattering amplitudes constructed as the sum of the tree graphs built from free propagators and (generally non-local) vertices derived from the interaction part of the non-local effective action $S_{\text {eff }}^{1-\text { loop }}\left[\phi_{\mathrm{cl}}\right]$. Note that these modified one-loop amplitudes differ from the original prescription because the latter one allows at most one vertex from $S_{\mathrm{CT}}^{1-\text { loop }}\left[\phi_{\mathrm{cl}}\right]+\Gamma_{\text {loop }}^{1-\text { loop }}\left[\phi_{\mathrm{cl}}\right]$ in each graph, while the former has no such a constraint.

As we have proved in the previous section, the effective action $S_{\mathrm{eff}}^{1-\text { loop }}\left[\phi_{\mathrm{cl}}\right]$ is invariant ${ }^{19}$ with respect to the hidden Special Galileon symmetry. Moreover, the effective action $S_{\text {eff }}^{1-\text { loop }}\left[\phi_{\mathrm{cl}}\right]$ contains only even vertices, especially there is no cubic vertex present. Therefore, using the general theorem connecting the symmetry of the action and the soft behavior of the tree-level scattering amplitudes [15], we can conclude that summing up all the tree graphs constructed with the use of $S_{\text {eff }}^{1-\text { loop }}\left[\phi_{\mathrm{cl}}\right]$, we get amplitudes with the enhanced $O\left(p^{3}\right)$ soft limit, i.e. the enhanced soft behavior is preserved also for the modified one-loop amplitudes.

Originally, we have been interested in the amplitudes given by graphs with index $i_{\mathrm{CT}}^{\Gamma} \leq D+2$. These correspond to a subset of graphs contributing to the modified oneloop scattering amplitudes, which can be identified by counting the powers of the scale $\Lambda$ introduced in (4.16) and (4.20), and keeping the contributions up to and including the order $O\left(\Lambda^{-D-2}\right)$. Here we have to treat the vertices stemming from $\Gamma_{\text {loop }}^{1-\text { loop }}\left[\phi_{\mathrm{cl}}\right]$ as $O\left(\Lambda^{-D+2}\right)$ (or with $i_{\mathrm{CT}}^{\Gamma}=D+2$ ).

\footnotetext{
${ }^{19}$ Or at least can be made invariant, provided we use invariant regularization as DR and if we allow only invariant finite counterterms in $S_{\mathrm{CT}, \text { finite }}^{1-\text { loop }}$ which we tacitly assume here.
} 
Because the contributions of the graphs with different $i_{\mathrm{CT}}^{\Gamma}$ cannot mutually cancel due to the different degree of homogeneity in momenta, this above soft theorem remains true also when we restrict ourselves to the contributions with $i_{\mathrm{CT}}^{\Gamma} \leq D+2$ only. In this sense, the soft theorem for the Special Galileon is valid also at the one-loop level.

\section{Special Galileon amplitudes at one loop - explicit example}

Let us illustrate the above considerations using the explicit expressions for the one-loop four-point amplitude in $D=4$. For definiteness, we will use the "plus" branch of the Special Galileon with the basic Lagrangian (1.3). The results for the "minus" branch can be obtained by the appropriate analytic continuation in the parameter $\alpha$.

The contribution $i_{\Gamma}=0$ corresponds to the basic Lagrangian and stems from the four-point vertex ${ }^{20}$

$$
\mathcal{L}_{\mathrm{b}}^{(4)}=\frac{1}{24 \alpha^{2}} \phi \varepsilon^{\mu_{1} \mu_{2} \mu_{3} \mu} \varepsilon^{\nu_{1} \nu_{2} \nu_{3} \nu} \eta_{\mu \nu} \prod_{i=1}^{3} \partial_{\mu_{i}} \partial_{\nu_{i}} \phi .
$$

The corresponding off-shell vertex in the momentum representation is

$$
V^{(4)}\left(p_{1}, p_{2}, p_{3}, p_{4}\right)=-\frac{1}{\alpha^{2}} G\left(p_{1}, p_{2}, p_{3}\right),
$$

where $G\left(p_{1}, p_{2}, p_{3}\right)$ is the Gramm determinant of the momenta $p_{1}, p_{2}$ and $p_{3}$. The on-shell amplitude reads then

$$
A^{i_{\Gamma}=0}=-\frac{1}{4 \alpha^{2}} s t u,
$$

where $s, t$ and $u$ are the usual Mandelstam variables

$$
\begin{aligned}
& s=\left(p_{1}+p_{2}\right)^{2}=\left(p_{3}+p_{4}\right)^{2} \\
& t=\left(p_{1}+p_{3}\right)^{2}=\left(p_{2}+p_{4}\right)^{2} \\
& u=\left(p_{1}+p_{4}\right)^{2}=\left(p_{2}+p_{3}\right)^{2},
\end{aligned}
$$

and all the momenta are treated as outgoing. The $O\left(p_{i}^{3}\right)$ soft behavior of the amplitude $A^{i_{\Gamma}=0}$ is manifest. The pure one-loop contributions correspond to the bubble graphs with two vertices (6.2), explicitly

$$
A_{1-\text { loop }}^{i_{\Gamma}=6}=\frac{1}{8} \sum_{\sigma \in S_{4}} A_{\text {bubble }}\left(p_{\sigma(1)}, p_{\sigma(2),} p_{\sigma(3),} p_{\sigma(4)}\right),
$$

where

$$
\begin{aligned}
& A_{\text {bubble }}\left(p_{i}, p_{j}, p_{k}, p_{l}\right)=\frac{1}{2}\left(\frac{1}{\alpha^{2}}\right)^{2}\left(p_{i} \cdot p_{j}\right)\left(p_{k} \cdot p_{l}\right) \\
& \quad \times \int \frac{\mathrm{d}^{d} l}{(2 \pi)^{d}} \frac{\left[2\left(p_{i} \cdot q_{i j}\right)\left(p_{j} \cdot q_{i j}\right)-q_{i j}^{2}\left(p_{i} \cdot p_{j}\right)\right]\left[2\left(p_{k} \cdot q_{k l}\right)\left(p_{l} \cdot q_{k l}\right)-q_{k l}^{2}\left(p_{k} \cdot p_{l}\right)\right]}{\left(q_{i j}^{2}+\mathrm{i} 0\right)\left(q_{k l}^{2}+\mathrm{i} 0\right)},
\end{aligned}
$$

\footnotetext{
${ }^{20}$ Note that we have to rescale the couplings (1.3) by a factor $-1 / 4$ ! in order to canonically normalize the kinetic term.
} 
and where $q_{m n}=l+\frac{1}{2}\left(p_{m}+p_{n}\right)$. For further convenience, let us also introduce the notation

$$
s_{i j}=s_{j i}=\left(p_{i}+p_{j}\right)^{2} .
$$

The result of the loop integration has the form

$$
A_{\text {bubble }}\left(p_{i}, p_{j}, p_{k}, p_{l}\right)=P\left(s_{i j}, s_{i k}, s_{i l} ; \varepsilon\right) B\left(s_{i j} ; \varepsilon\right),
$$

where $\varepsilon=2-d / 2$, the function $B\left(s_{i j}\right)$ is the scalar two-point function given as

$$
\begin{aligned}
B\left(s_{i j}\right) & =-\mathrm{i} \int \frac{\mathrm{d}^{d} l}{(2 \pi)^{d}} \frac{1}{\left[q_{i j}^{2}+\mathrm{i} 0\right]\left[q_{k l}^{2}+\mathrm{i} 0\right]}=\frac{\mu^{-2 \varepsilon}}{(4 \pi)^{2-\varepsilon}} \frac{\Gamma(\varepsilon) \Gamma(1-\varepsilon)^{2}}{\Gamma(2-2 \varepsilon)}\left(-\frac{s_{i j}}{\mu^{2}}\right)^{-\varepsilon} \\
& =\frac{\mu^{-2 \varepsilon}}{(4 \pi)^{2}}\left[\frac{1}{\varepsilon}-\gamma+\ln 4 \pi+2-\ln \left(-\frac{s_{i j}}{\mu^{2}}\right)+O(\varepsilon)\right]
\end{aligned}
$$

and $P$ is the following polynomial

$$
P\left(s_{i j}, s_{i k}, s_{i l} ; \varepsilon\right)=\frac{1}{512 \alpha^{4}\left(d^{2}-1\right)} s_{i j}^{4}\left[s_{i j}^{2} d(d-2)-8 s_{i k} s_{i l}\right] .
$$

Finally, we get for the bubble

$$
\begin{aligned}
A_{\text {bubble }}\left(p_{i}, p_{j}, p_{k}, p_{l}\right)= & \frac{\mu^{-2 \varepsilon}}{(4 \pi)^{2}} \frac{s_{i j}^{4}}{1920 \alpha^{4}} \\
& \times\left\{\left(s_{i j}^{2}+s_{i k}^{2}+s_{i l}^{2}\right)\left[\frac{1}{\varepsilon}+c_{\text {bubble }}-\ln \left(-\frac{s_{i j}}{\mu^{2}}\right)\right]-3 s_{i j}^{2}\right\} .
\end{aligned}
$$

The actual value of the constant $c_{\text {bubble }}$ depends on the details of the dimensional regularization scheme. We use the t'Hooft-Veltman scheme for the reduction of the tensor integrals for which

$$
c_{\text {bubble }}=-\gamma+\ln 4 \pi+\frac{46}{15} .
$$

The complete one-loop contribution to the amplitude is then

$$
\begin{aligned}
A_{1-\text { loop }}^{i_{\Gamma}=6}= & \frac{\mu^{-2 \varepsilon}}{(4 \pi)^{2}} \frac{1}{1920 \alpha^{4}}\left\{\left(\frac{1}{\varepsilon}+c_{\text {bubble }}\right) \frac{1}{2}\left(s^{2}+t^{2}+u^{2}\right)^{3}\right. \\
& -\left(s^{2}+t^{2}+u^{2}\right)\left[s^{4} \ln \left(-\frac{s}{\mu^{2}}\right)+t^{4} \ln \left(-\frac{t}{\mu^{2}}\right)+u^{4} \ln \left(-\frac{u}{\mu^{2}}\right)\right] \\
& \left.-3\left(s^{6}+t^{6}+u^{6}\right)\right\} .
\end{aligned}
$$

Let us now consider the contributions of the higher derivative counterterms. At the level $i_{\Gamma}=2$, we have

$$
\begin{aligned}
c_{4} \frac{\alpha^{2}}{\Lambda^{2}} \sqrt{g}= & c_{4} \frac{\alpha^{2}}{\Lambda^{2}}\left[1+\frac{1}{2 \alpha^{2}}\langle\partial \partial \phi . \partial \partial \phi\rangle\right. \\
& \left.-\frac{1}{4 \alpha^{4}}\langle\partial \partial \phi . \partial \partial \phi . \partial \partial \phi . \partial \partial \phi\rangle+\frac{1}{8 \alpha^{4}}\langle\partial \partial \phi . \partial \partial \phi\rangle^{2}+O\left(\phi^{6}\right)\right],
\end{aligned}
$$


where now the normal dot (and normal colon in what follows) means a contraction of adjacent indices with the flat metric $\eta^{\alpha \beta}$ and $\langle\cdot\rangle$ denotes the trace with respect to the same flat metric. The effect of this Lagrangian is therefore twofold. The term quadratic in the fields contributes to the off-shell two point function of the field $\phi$; for this contribution, we get explicitly

$$
\Sigma^{i_{\Gamma}=2}\left(p^{2}\right)=-\frac{c_{4}}{\Lambda^{2}} p^{4} .
$$

Note, however, that the derivative of $\Sigma^{i_{\Gamma}}=2\left(p^{2}\right)$ vanishes on shell, and therefore there is no external leg renormalization. The quartic terms in (6.14) are responsible for the contact contribution to the four-point on-shell amplitude. We get

$$
A_{\mathrm{CT}}^{i_{\Gamma}=2}=-\frac{c_{4}}{\Lambda^{2}} \frac{1}{128 \alpha^{2}} \sum_{\sigma \in S_{4}} s_{\sigma(1) \sigma(2)}^{2}\left[s_{\sigma(1) \sigma(4)}^{2}-\frac{1}{2} s_{\sigma(1) \sigma(2)}^{2}\right]=0,
$$

and thus there is no $i_{\Gamma}=2$ contribution to the four-point amplitude. At the next level $i_{\Gamma}=4$, we have the following expansion of the Lagrangian

$$
\begin{aligned}
c_{3} \frac{\alpha^{2}}{\Lambda^{4}} \sqrt{g} R= & -\frac{c_{3}}{\Lambda^{4}}\left[\langle\partial \partial \partial \phi: \partial \partial \partial \phi\rangle-\frac{3}{\alpha^{2}}\langle\partial \partial \partial \phi: \partial \partial \partial \phi . \partial \partial \phi . \partial \partial \phi\rangle\right. \\
& \left.+\frac{1}{2 \alpha^{2}}\langle\partial \partial \phi . \partial \partial \phi\rangle\langle\partial \partial \partial \phi: \partial \partial \partial \phi\rangle+O\left(\phi^{6}\right)\right] .
\end{aligned}
$$

Again, there is a contribution to the off-shell two point function, which, however, has no effect on the on-shell four-point amplitude. The contact contribution which stems from the quartic term gives

$$
\begin{aligned}
A_{\mathrm{CT}}^{i_{\Gamma}=4} & =-\frac{c_{3}}{\Lambda^{4}} \frac{3}{32 \alpha^{2}} \sum_{\sigma \in S_{4}} s_{\sigma(1) \sigma(2)}^{3}\left[s_{\sigma(1) \sigma(4)}^{2}-\frac{1}{6} s_{\sigma(1) \sigma(2)}^{2}\right] \\
& =\frac{c_{3}}{\Lambda^{4}} \frac{1}{20 \alpha^{2}}\left(s^{5}+t^{5}+u^{5}\right) .
\end{aligned}
$$

Finally, for the $i_{\Gamma}=4$ counterterm contribution, we have the Lagrangian

$$
\begin{aligned}
\mu^{-2 \varepsilon} \frac{\alpha^{2}}{\Lambda^{6}} \sqrt{g}\left(c_{1} R_{\mu \nu} R^{\mu \nu}+c_{2} R^{2}\right)= & \frac{\mu^{-2 \varepsilon}}{\Lambda^{6}} \frac{1}{\alpha^{2}}\left[c_{1}\langle\partial \partial \partial \phi: \partial \partial \partial \phi . \partial \partial \partial \phi: \partial \partial \partial \phi\rangle\right. \\
& \left.+c_{2}\langle\partial \partial \partial \phi: \partial \partial \partial \phi\rangle^{2}+O\left(\phi^{6}\right)\right],
\end{aligned}
$$

which yields the contact terms of the form

$$
\begin{aligned}
A_{\mathrm{CT}}^{i_{\Gamma}=6} & =\frac{\mu^{-2 \varepsilon}}{\Lambda^{6}} \frac{1}{64 \alpha^{2}} \sum_{\sigma \in S_{4}} s_{\sigma(1) \sigma(2)}^{4}\left[c_{1} s_{\sigma(1) \sigma(4)}^{2}+c_{2} s_{\sigma(1) \sigma(2)}^{2}\right] \\
& =\frac{\mu^{-2 \varepsilon}}{\Lambda^{6}} \frac{1}{8 \alpha^{2}}\left[\frac{1}{4} c_{1}\left(s^{2}+t^{2}+u^{2}\right)^{3}+\left(c_{2}-\frac{1}{2} c_{1}\right)\left(s^{6}+t^{6}+u^{6}\right)\right] .
\end{aligned}
$$

The infinite part of $A_{\mathrm{CT}}^{i_{\Gamma}=6}$ then reads

$$
A_{\mathrm{CT}, \infty}^{i_{\Gamma}=6}=-\frac{\mu^{-2 \varepsilon}}{(4 \pi)^{2}} \frac{1}{\varepsilon} \frac{1}{3840 \alpha^{4}}\left(s^{2}+t^{2}+u^{2}\right)^{3}
$$


and cancels the infinite part of the of the loop contribution $A_{1-\text { loop }}^{i_{\Gamma}=6}$. The finite part of $A_{\mathrm{CT}}^{i_{\Gamma}=6}$ can be obtained from (6.20) by replacing $c_{i} \rightarrow c_{i}^{\mathrm{r}}(\mu)$. Finally, we get ${ }^{21}$

$$
\begin{aligned}
A^{i_{\Gamma} \leq 6}= & -\frac{1}{4 \alpha^{2}} s t u+\frac{1}{\Lambda^{4}} \frac{c_{3}^{\mathrm{r}}(\mu)}{20 \alpha^{2}}\left(s^{5}+t^{5}+u^{5}\right)+\frac{1}{\Lambda^{6}}\left(\frac{k_{1}^{\mathrm{r}}(\mu)}{8 \alpha^{2}} s^{2} t^{2} u^{2}+\frac{k_{2}^{\mathrm{r}}(\mu)}{8 \alpha^{2}}\left(s^{6}+t^{6}+u^{6}\right)\right) \\
& -\frac{1}{(4 \pi)^{2}} \frac{1}{1920 \alpha^{4}}\left\{\left(s^{2}+t^{2}+u^{2}\right)\left[s^{4} \ln \left(-\frac{s}{\mu^{2}}\right)+t^{4} \ln \left(-\frac{t}{\mu^{2}}\right)+u^{4} \ln \left(-\frac{u}{\mu^{2}}\right)\right]\right\},
\end{aligned}
$$

where we have abbreviated

$$
\begin{aligned}
& k_{1}^{\mathrm{r}}(\mu)=-3 c_{1}^{\mathrm{r}}(\mu)-\frac{\Lambda^{6}}{(4 \pi)^{2}} \frac{1}{40 \alpha^{2}}\left(-\gamma+\ln 4 \pi+\frac{46}{15}\right) \\
& k_{2}^{\mathrm{r}}(\mu)=c_{2}^{\mathrm{r}}(\mu)+\frac{1}{2} c_{1}^{\mathrm{r}}(\mu)+\frac{\Lambda^{6}}{(4 \pi)^{2}} \frac{1}{120 \alpha^{2}}\left[\left(-\gamma+\ln 4 \pi+\frac{46}{15}\right)-\frac{3}{2}\right] .
\end{aligned}
$$

Note that the $O\left(p^{3}\right)$ soft behavior is manifest for all the above components of the amplitude. In the case of the four-point amplitude, it is somewhat trivial statement due to the special four-particle kinematics and due to the power counting of the individual contributions.

\section{Summary and discussion}

In this paper, we have studied the issue of the one-loop renormalization of the Special Galileon $S$-matrix. First, we have calculated the UV divergent part $\Gamma_{\text {loop }}^{1-\text { loop }}\left[\phi_{\mathrm{cl}}\right]_{\infty}$ of the one-loop on-shell effective action and proved its invariance with respect to the hidden Special Galileon symmetry for a general space-time dimension $D$. The key ingredient of the proof was the fact that we were able to express the action describing the quantum fluctuations in the on-shell classical background in terms of the geometric building blocks which were covariant with respect to the Special Galileon symmetry. We have further found the appropriate prescription for the transformation of the fluctuating field with respect to the Special Galileon symmetry which ensures the invariance of the fluctuation action and, as a consequence, also the invariance of the complete one-loop on-shell effective action $\Gamma_{\text {loop }}^{1-\text { loop }}\left[\phi_{\mathrm{cl}}\right]$.

For the $D=4$ case, we have constructed the complete set of independent higher derivative counterterms relevant for the calculation of the scattering amplitudes, up to and including graphs with index $i_{\Gamma}=D_{\Gamma}-2 E_{\Gamma}+2=6$. The resulting counterterm action can be expressed in a manifestly invariant form with respect to the hidden Special Galileon symmetry, namely

$$
S_{\mathrm{CT}}^{1-\text { loop }}=\mu^{-2 \varepsilon} \int \mathrm{d}^{4} x \sqrt{g} \sum_{j=1}^{4} \frac{\alpha^{2}}{\Lambda^{i_{\mathcal{O}_{j}}}} c_{j} \mathcal{O}_{j} .
$$

\footnotetext{
${ }^{21}$ Here we have used the identity

$$
\left(s^{2}+t^{2}+u^{2}\right)^{3}=-12 s^{2} t^{2} u^{2}+4\left(s^{6}+t^{6}+u^{6}\right)
$$
}

in order to be able to compare the results of the section 6 with the existing literature. 
Here $\mathrm{d}^{4} x \sqrt{g}$ is the invariant measure corresponding to the effective metric

$$
g_{\mu \nu}=\eta_{\mu \nu} \pm \frac{1}{\alpha^{2}} \partial_{\mu} \partial_{\alpha} \phi \partial^{\alpha} \partial_{\nu} \phi
$$

and the invariant operators $\mathcal{O}_{j}$ are given in terms of the extrinsic curvature tensor

$$
\mathcal{K}_{\alpha \mu \nu}=-\frac{1}{\alpha} \partial_{\alpha} \partial_{\mu} \partial_{\nu} \phi
$$

and in terms of the inverse effective metric $g^{\mu \nu}$ as

$$
\begin{aligned}
& \mathcal{O}_{1}=\langle\langle\mathcal{K}: \mathcal{K} \cdot \mathcal{K}: \mathcal{K}\rangle\rangle \\
& \mathcal{O}_{2}=\langle\langle\mathcal{K}: \mathcal{K}\rangle\rangle^{2} \\
& \mathcal{O}_{3}=\langle\langle\mathcal{K}: \mathcal{K}\rangle\rangle \\
& \mathcal{O}_{4}=1 .
\end{aligned}
$$

Here the bold dots and $\langle\langle\ldots\rangle\rangle$ mean contractions and the trace with respect to $g^{\mu \nu}$ respectively. These operators form a complete basis of the on-shell counterterms relevant for the renormalization of the $S$-matrix at one loop and $i_{\Gamma} \leq 6$. We have identified the infinite parts of the bare coupling $c_{j}$ and established the running of the corresponding renormalized couplings $c_{j}^{\mathrm{r}}(\mu)$ with the renormalization scale $\mu$.

Note that the action $S_{\mathrm{CT}}^{1-\text { loop }}$ generates only even vertices (especially, there is no cubic vertex present). As we have proved, also the non-local part of the $i_{\Gamma} \leq 6$ on-shell effective action $S_{\text {eff }}^{1-\text { loop }}$ stemming from the loops is invariant with respect to the hidden Special Galileon symmetry and contains only even non-local vertices. Using the general theorem about the relation between generalized polynomial shift symmetries and the soft behavior of the scattering amplitudes, we have proved that the enhanced $O\left(p^{3}\right)$ soft limit of the amplitudes is preserved also at one loop and $i_{\Gamma} \leq 6$.

Let us stress that the proof of the manifest invariance of $S_{\mathrm{CT}}^{1-\text { loop }}$ and of the nonlocal one-loop effective action $S_{\text {eff }}^{1-\text { loop }}$ heavily depends on the fact that only the on-shell configurations satisfying the classical equation of motion derived from the basic Lagrangians are relevant for the calculation of the on-shell scattering amplitudes. Also, this classical equation of motion was used for the elimination of the redundant operators and reduction of the basis of the counterterms. Therefore, though the counterterm action $S_{\mathrm{CT}}^{1-\text { loop }}$ guarantees the finiteness of the on-shell scattering amplitudes, it is not sufficient to make also the offshell Green functions finite.

In order to renormalize also the one-loop off-shell Green functions, no simple explicit calculation of the UV divergent part of the effective action is possible. In general, we can expect that additional counterterms will be needed, and that the simple structure of the higher order Lagrangians will be lost. For instance, the most general counterterm action invariant with respect to the hidden Special Galileon symmetry should be of the form (1.21) with an additional invariant odd in the field which vanish on-shell, namely ${ }^{22}$

$$
\sigma=\frac{\alpha}{2 \mathrm{i}} \ln \left[\frac{\operatorname{det}\left(\eta+\frac{\mathrm{i}}{\alpha} \partial \partial \phi\right)}{\operatorname{det}\left(\eta-\frac{\mathrm{i}}{\alpha} \partial \partial \phi\right)}\right] .
$$

\footnotetext{
${ }^{22}$ Here we present the formula for the "plus" branch of the transformation (1.5). The "minus" branch variant can be obtained with the replacement $\alpha \rightarrow \mathrm{i} \alpha$.
} 
Naively, at the level $i_{\Gamma} \leq 6$, we might expect the invariant action of the form

$$
S_{\mathrm{CT}}=\mu^{-2 \varepsilon} \int \mathrm{d}^{4} x \sqrt{g} \sum_{j} \frac{\alpha^{2}}{\Lambda^{i_{\mathcal{O}_{j}}}} c_{j} \mathcal{O}_{j},
$$

where the diffeomorphism invariant operators $\mathcal{O}_{j}$ are now constructed as contractions (with respect to the effective metric $g^{\mu \nu}$ ) of the extrinsic curvature tensor, its covariant derivatives, and covariant derivatives of the scalar $\sigma$. Schematically

$$
\mathcal{O}_{j} \sim\left(D^{n_{j}} \mathcal{K}^{m_{j}}\right)\left(D^{r_{j}} \sigma\right)^{k_{j}}
$$

with $n_{j}+m_{j}+r_{j} k_{j} \leq 4$ (see e.g. the operators $\mathcal{O}_{5}, \ldots, \mathcal{O}_{8}$ discussed in section 4 and appendix A). Listing a complete basis of such off-shell independent operators is beyond the scope of this paper. However, even if we were able to classify the operators $\mathcal{O}_{j}$ of the above type, this is not the whole story. Note that the constants $c_{j}$ in (7.6) can be freely replaced with arbitrary functions $c_{j}(\sigma)$ without changing the index of the corresponding counterterms. Thus, the presence of the off-shell invariant $\sigma$ obscures the complete classification of the off-shell counterterm action $S_{\mathrm{CT}}$. The situation is somewhat similar to the three-flavor chiral perturbation theory with the additional $U(1)$ pseudoscalar corresponding to $\eta^{\prime}$ (cf. $[23,24]$ ). In that case, the invariant Lagrangian is determined up to arbitrary potentials which are functions of the $\eta^{\prime}$ field. Note also that because the off-shell invariant $\sigma$ is odd in the field, the off-shell basis should also contain the operators giving rise to the odd off-shell vertices. However, the contributions of such vertices have to cancel mutually in the on-shell amplitudes.

Recently, a complementary classification of the higher order Special Galileon Lagrangians in $D=4$ has been developed using the coset construction based on the Special Galileon symmetry algebra $[25,26]$. The basic building blocks of this constructions are the invariant measure $\mathrm{d}^{4} x \operatorname{det}(E)$, the covariant derivative $\nabla_{a} \xi^{a}$ of the Goldstone field $\xi^{a}$ corresponding to the linear part of the general Galileon symmetry (1.1), and the covariant derivative $\nabla_{c} \sigma_{a b}$ of the Goldstone field $\sigma_{a b}$ corresponding to the hidden Special Galileon symmetry $^{23}(1.5)$. The Latin indices are then contracted with the flat metric $\eta^{a b}$ to build the invariants. Though the direct comparison of these building blocks with our approach is difficult, we have found the following correspondence with our geometrical objects valid up to denoted higher orders in the field $\phi$

$$
\begin{aligned}
\operatorname{det}(E) & =\sqrt{g}\left(1-\frac{1}{8}\left(\frac{\sigma}{\alpha}\right)^{2}+\frac{5}{768}\left(\frac{\sigma}{\alpha}\right)^{4}-\frac{17}{92160}\left(\frac{\sigma}{\alpha}\right)^{6}\right)+O\left(\phi^{8}\right) \\
\nabla_{a} \xi^{a} & =-\sigma\left(1+\frac{1}{48}\left(\frac{\sigma}{\alpha}\right)^{2}+\frac{1}{1920}\left(\frac{\sigma}{\alpha}\right)^{4}\right)+O\left(\phi^{7}\right) \\
\nabla_{c} \sigma_{a b} & =\left(\mathcal{K}_{c a b}-\frac{1}{4} \eta_{a b} \mathcal{K}_{c \mu \nu} g^{\mu \nu}\right)\left(1+\frac{1}{32}\left(\frac{\sigma}{\alpha}\right)^{2}\right)+O\left(\phi^{5}\right),
\end{aligned}
$$

where

$$
\mathcal{K}_{c a b}=m_{a}^{\mu} m_{b}^{\nu} m_{c}^{\alpha} \mathcal{K}_{\alpha \mu \nu}, \mathcal{K}_{c \mu \nu}=m_{c}^{\alpha} \mathcal{K}_{\alpha \mu \nu},
$$

\footnotetext{
${ }^{23}$ The authors restrict themselves to the "plus" branch and their $\alpha$ parameter corresponds to our $1 / \alpha$.
} 
and where

$$
m_{a}^{\mu}=\delta_{a}^{\mu}+\sum_{n=1}^{\infty} \frac{(1 / 2-n)_{n}}{n !} \frac{1}{\alpha^{2 n}}\left[(\boldsymbol{\partial} \boldsymbol{\partial} \phi)^{2 n}\right]_{a}^{\mu}
$$

is the vielbein for the inverse effective metric $g^{\mu \nu}=\eta^{a b} m_{a}^{\mu} m_{b}^{\nu}$ mentioned in the introduction (in a particular gauge, see [21] for more details). We can thus conclude that on shell and up to the higher orders mentioned above

$$
\operatorname{det}(E)=\sqrt{g}, \quad \nabla_{a} \xi^{a}=0, \quad \nabla_{c} \sigma_{a b}=\mathcal{K}_{c a b} .
$$

Therefore, the building blocks for the on-shell higher derivative action are the same as in our geometrical approach, at least for the vertices necessary for the calculation of the $4 \mathrm{pt}$, 5 pt and $6 \mathrm{pt}$ scattering amplitudes at $i_{\Gamma} \leq 6$. This especially means that there is no cubic and quintic vertex relevant for the on-shell amplitudes. Though such vertices might be present in the off-shell higher derivative action, e.g. in the Lagrangian introduced in [26] as

$$
\mathcal{L}_{\text {odd }}^{i_{\Gamma}=2}=\operatorname{det}(E) \Delta \mathcal{L}^{(0)}=\operatorname{det}(E) a_{1} \nabla_{a} \xi^{a}
$$

their contribution have to vanish on shell. As a consequence, the leading order $O\left(p^{10}\right) 5 \mathrm{pt}$ amplitude proportional to $a_{1}$ as well as the $a_{1}^{2}$ contribution to the $4 \mathrm{pt}$ amplitude have to vanish on shell.

As an illustration of the one-loop renormalization of the Special Galileon, we have calculated the four-particle amplitude up to and including the contributions with $i_{\Gamma}=6$. The result reads

$$
\begin{aligned}
A_{4}^{i_{\Gamma} \leq 6}= & -\frac{1}{4 \alpha^{2}} s t u+\frac{1}{\Lambda^{4}} \frac{c_{3}^{\mathrm{r}}(\mu)}{20 \alpha^{2}}\left(s^{5}+t^{5}+u^{5}\right)+\frac{1}{\Lambda^{6}}\left(\frac{k_{1}^{\mathrm{r}}(\mu)}{8 \alpha^{2}} s^{2} t^{2} u^{2}+\frac{k_{2}^{\mathrm{r}}(\mu)}{8 \alpha^{2}}\left(s^{6}+t^{6}+u^{6}\right)\right) \\
& -\frac{1}{(4 \pi)^{2}} \frac{1}{1920 \alpha^{4}}\left\{\left(s^{2}+t^{2}+u^{2}\right)\left[s^{4} \ln \left(-\frac{s}{\mu^{2}}\right)+t^{4} \ln \left(-\frac{t}{\mu^{2}}\right)+u^{4} \ln \left(-\frac{u}{\mu^{2}}\right)\right]\right\},
\end{aligned}
$$

where $c_{j}^{\mathrm{r}}(\mu)$ and $k_{j}^{\mathrm{r}}(\mu)$ are (linear combinations of) the couplings ${ }^{24}$ renormalized at the scale $\mu$. The running of these couplings with $\mu$ given by (4.32), and (4.31) ensures the manifest renormalization scale independence of $A_{4}^{i_{\Gamma} \leq 6}$. This amplitude satisfies also manifestly the soft theorem with the $O\left(p^{3}\right)$ soft behavior. Let us note that the polynomial part of $A_{4}^{i_{\Gamma} \leq 6}$ has been determined independently in [26] from the Lagrangian constructed using the coset formalism with the same result up to a redefinition of the couplings. Alternative determination of the polynomial part was presented in [41] using the soft bootstrap with the conclusion that the consistency of the soft BCFW recursion for the tree-level six-particle amplitude forces the constant $k_{2}^{\mathrm{r}}(\mu)$ to vanish. This conclusion was supported by the KLT double copy construction of the amplitude. However, even if we set $k_{2}^{\mathrm{r}}(\mu)=0$ at some scale due to the running of $k_{2}^{\mathrm{r}}(\mu)$, such a term is inevitably generated by the loop corrections at other scale $\mu^{\prime}$.

\footnotetext{
${ }^{24}$ See (6.23) for explicit formulas.
} 
The next five-particle amplitude with $i_{\Gamma} \leq 6$ vanishes since there are no odd vertices in the one-loop on-shell effective action $S_{\text {eff }}^{1-\text { loop }}$

$$
A_{5}^{i_{\Gamma} \leq 6}=0 .
$$

Note that in [41], there has been a 5 pt amplitude with $i_{\Gamma}=6$ constructed consistent with the $O\left(p^{3}\right)$ soft behavior, namely

$$
\begin{aligned}
A_{5}^{i_{\Gamma}=6}= & \frac{c_{5}}{\Lambda^{6} \alpha^{3}} \varepsilon_{\mu_{1} \mu_{2} \mu_{3} \mu_{4}} \sum_{\sigma \in S_{5}} p_{\sigma(1)}^{\mu_{1}} p_{\sigma(2)}^{\mu_{2}} p_{\sigma(3)}^{\mu_{3}} p_{\sigma(4)}^{\mu_{4}} \\
& \times\left(p_{\sigma(1)} \cdot p_{\sigma(2)}\right)\left(p_{\sigma(2)} \cdot p_{\sigma(3)}\right)\left(p_{\sigma(3)} \cdot p_{\sigma(4)}\right)\left(p_{\sigma(4)} \cdot p_{\sigma(5)}\right)\left(p_{\sigma(5)} \cdot p_{\sigma(1)}\right) .
\end{aligned}
$$

Though the soft bootstrap probe does not exclude such an amplitude, it has been established that it cannot be obtained by the KLT double copy construction. In our approach, such an amplitude is excluded since it cannot stem from any operator invariant with respect to the Special Galileon symmetry.

Our results therefore support the conjecture that symmetry based definition of the Special Galileon is in tension with the KLT double copy construction at the higher orders as claimed in [26]. However, we have not confirmed the violation of the interrelation between the Special Galileon symmetry and the $O\left(p^{3}\right)$ soft behavior of the amplitudes since we have proved that at least for $i_{\Gamma} \leq 6$, there does not exist any cubic vertex relevant for the on-shell amplitudes.

\section{A Classification of the one loop on-shell counterterms}

In this appendix, we prove some statements concerning the elimination of redundant onshell counterterms. Let us first remind the Codazzi equation for the extrinsic curvature

$$
D_{\mu} K_{\nu \rho}^{a}-D_{\nu} K_{\mu \rho}^{a}=0
$$

which is valid in our case due to the geometrical interpretation of the Special Galileon field as an effective theory of the $D$-dimensional brane in a flat $2 D$-dimensional target space [21]. This can be rewritten as a symmetry relation for the covariant derivative of the tensor $\mathcal{K}_{\alpha \mu \nu}=g_{\alpha \beta} m_{a}^{\beta} K_{\mu \nu}^{a}$, where $m_{a}^{\beta}$ is the $D$-bein for the induced metric $g^{\mu \nu}$, as ${ }^{25}$

$$
D_{\mu} \mathcal{K}_{\alpha \nu \rho}=D_{\nu} \mathcal{K}_{\alpha \mu \rho}
$$

As a consequence,

$$
(D \cdot \mathcal{K})_{\nu \rho}=g^{\mu \alpha} D_{\mu} \mathcal{K}_{\alpha \nu \rho}=g^{\mu \alpha} D_{\nu} \mathcal{K}_{\alpha \mu \rho}=D_{\nu} g^{\mu \alpha} \mathcal{K}_{\alpha \mu \rho}=0,
$$

where the last equation holds on shell as a consequence of (4.11). Therefore the operators

$$
\begin{aligned}
\mathcal{O}_{5} & =\langle\langle(\mathcal{K}: \mathcal{K}) \cdot(D \cdot \mathcal{K})\rangle\rangle, \\
\mathcal{O}_{6} & =(D \cdot \mathcal{K}):(D \cdot \mathcal{K}),
\end{aligned}
$$

\footnotetext{
${ }^{25}$ Note that $g_{\alpha \beta}$ and $m_{a}^{\mu}$ are covariantly constant.
} 
vanish on shell. Similarly, with the help of (A.2) and (4.11), we get on shell

$$
\begin{aligned}
(D \cdot D) \mathcal{K}_{\alpha \beta \rho} & =g^{\mu \nu} D_{\mu} D_{\nu} \mathcal{K}_{\alpha \beta \rho}=g^{\mu \nu} D_{\mu} D_{\beta} \mathcal{K}_{\alpha \nu \rho}=g^{\mu \nu}\left[D_{\mu}, D_{\beta}\right] \mathcal{K}_{\alpha \nu \rho}+D_{\beta} g^{\mu \nu} D_{\mu} \mathcal{K}_{\alpha \nu \rho} \\
& =g^{\mu \nu}\left[D_{\mu}, D_{\beta}\right] \mathcal{K}_{\alpha \nu \rho}=g^{\mu \nu}\left(R_{\alpha \beta \mu}^{\sigma} \mathcal{K}_{\sigma \nu \rho}+R_{\nu \beta \mu}^{\sigma} \mathcal{K}_{\alpha \sigma \rho}+R_{\rho \beta \mu}^{\sigma} \mathcal{K}_{\alpha \nu \sigma}\right) \\
& =g^{\mu \nu} g^{\sigma \kappa}\left(R_{\kappa \alpha \beta \mu} \mathcal{K}_{\sigma \nu \rho}+R_{\kappa \nu \beta \mu} \mathcal{K}_{\alpha \sigma \rho}+R_{\kappa \rho \beta \mu} \mathcal{K}_{\alpha \nu \sigma}\right)
\end{aligned}
$$

Now, using the Gauss equation for the Riemann tensor

$$
R_{\kappa \alpha \beta \mu}=\mathcal{K}_{\kappa \beta} \cdot \mathcal{K}_{\alpha \mu}-\mathcal{K}_{\kappa \mu} \cdot \mathcal{K}_{\alpha \beta},
$$

and after some algebra, using the total symmetry of $\mathcal{K}_{\alpha \mu \rho}$ and (4.11), we have

$$
\begin{aligned}
(D \cdot D) \mathcal{K}_{\alpha \beta \rho}= & 2 g^{\mu \nu} \mathcal{K}_{\nu \rho} \cdot \mathcal{K}_{\beta} \cdot \mathcal{K}_{\alpha \mu} \\
& -\mathcal{K}_{\rho}: \mathcal{K} \cdot \mathcal{K}_{\alpha \beta}-\mathcal{K}_{\beta}: \mathcal{K} \cdot \mathcal{K}_{\alpha \rho}-\mathcal{K}_{\alpha}: \mathcal{K} \cdot \mathcal{K}_{\rho \beta} .
\end{aligned}
$$

Finally, we get for the operator $\mathcal{O}_{7}$

$$
\mathcal{O}_{7}=\langle\langle(\mathcal{K}:(D \cdot D) \mathcal{K})\rangle\rangle=2 \mathcal{O}_{8}-3 \mathcal{O}_{1},
$$

where $\mathcal{O}_{1}$ and $\mathcal{O}_{8}$ are defined as

$$
\begin{aligned}
& \mathcal{O}_{1}=\langle\langle\mathcal{K}: \mathcal{K} \cdot \mathcal{K}: \mathcal{K}\rangle\rangle=R_{\mu \nu} R^{\mu \nu} \\
& \mathcal{O}_{8}=g^{\kappa \rho} g^{\gamma \beta} g^{\delta \alpha} g^{\mu \nu} \mathcal{K}_{\kappa \gamma \delta} \mathcal{K}_{\nu \rho} \cdot \mathcal{K}_{\beta} \cdot \mathcal{K}_{\alpha \mu} .
\end{aligned}
$$

As far as the operator $\mathcal{O}_{8}$ term is concerned, let us remind that

$$
\begin{aligned}
R_{\kappa \alpha \beta \mu} R^{\kappa \alpha \beta \mu} & =2\langle\langle\mathcal{K}: \mathcal{K} \cdot \mathcal{K}: \mathcal{K}\rangle\rangle-2 g^{\kappa \rho} g^{\gamma \beta} g^{\delta \alpha} g^{\mu \nu} \mathcal{K}_{\kappa \gamma \delta} \mathcal{K}_{\nu \rho} \cdot \mathcal{K}_{\beta} \cdot \mathcal{K}_{\alpha \mu} \\
& =2 \mathcal{O}_{1}-2 \mathcal{O}_{8}
\end{aligned}
$$

and thus the operators $\mathcal{O}_{7}$ and $\mathcal{O}_{8}$ can be expressed in terms of the squares of the Riemann and Ricci tensor as

$$
\begin{aligned}
& \mathcal{O}_{7}=-R_{\kappa \alpha \beta \mu} R^{\kappa \alpha \beta \mu}-R_{\mu \nu} R^{\mu \nu}, \\
& \mathcal{O}_{8}=R_{\mu \nu} R^{\mu \nu}-\frac{1}{2} R_{\kappa \alpha \beta \mu} R^{\kappa \alpha \beta \mu} .
\end{aligned}
$$

Therefore, up to a total derivative corresponding to the Gauss-Bonnet term $G$ (cf. (4.8)), these operators are linear combinations of $\mathcal{O}_{1}$ and $\mathcal{O}_{2}$ (see (4.17) and (4.18))

$$
\begin{aligned}
& \mathcal{O}_{7}=-5 R_{\mu \nu} R^{\mu \nu}+R^{2}-G=-5 \mathcal{O}_{1}+\mathcal{O}_{2}-G, \\
& \mathcal{O}_{8}=-R_{\mu \nu} R^{\mu \nu}+\frac{1}{2} R^{2}-\frac{1}{2} G=-\mathcal{O}_{1}+\frac{1}{2} \mathcal{O}_{2}-\frac{1}{2} G .
\end{aligned}
$$

Let us now concentrate on the parity odd operators

$$
\mathcal{O}_{j}=\left(D^{n_{j}} \mathcal{K}^{m_{j}}\right)_{\mu_{1} \mu_{2} \mu_{3} \mu_{4}} E^{\mu_{1} \mu_{2} \mu_{3} \mu_{4}},
$$

where $n_{j}+m_{j} \leq 4$ and where

$$
E^{\mu_{1} \ldots \mu_{D}}=\frac{1}{\sqrt{g}} \varepsilon^{\mu_{1} \ldots \mu_{D}}
$$


Note that, due to the symmetry of $\mathcal{K}_{\alpha \mu \nu}$, within the tensor $\left(D^{n_{j}} \mathcal{K}^{m_{j}}\right)_{\mu_{1} \mu_{2} \mu_{3} \mu_{4}}$ each $\mathcal{K}$ can have at least one uncontracted index. Also, $g^{\mu \nu} \mathcal{K}_{\alpha \mu \nu}=0$ on shell so that only indices within different $\mathcal{K}$ 's can be contracted.

There are no such $i_{\Gamma}=2$ and $i_{\Gamma}=4$ operators since there are not enough contractions to satisfy the above requirements. For instance, for $i_{\Gamma}=4$, the following possible operator with $n_{j}=0, m_{j}=2$ vanishes

$$
\mathcal{O}_{9}^{i_{\Gamma}=4}=\left(\mathcal{K}_{\mu_{1} \mu_{2}} \cdot \mathcal{K}_{\mu_{3} \mu_{4}}\right) E^{\mu_{1} \mu_{2} \mu_{3} \mu_{4}}=0
$$

since we have to contract the extrinsic curvature tensors $\mathcal{K}_{\alpha \mu \nu}$ once, however the result of this contraction is symmetric in $\mu_{1} \mu_{2}$ and $\mu_{3} \mu_{4}$. Similarly, because $D \cdot \mathcal{K}_{\mu \nu}=0$ on shell, the possible operator with $n_{j}=1, m_{j}=1$

$$
\mathcal{O}_{10}^{i_{\Gamma}=4}=D_{\mu_{1}} \mathcal{K}_{\mu_{2} \mu_{3} \mu_{4}} E^{\mu_{1} \mu_{2} \mu_{3} \mu_{4}}=0 .
$$

At the next level ${ }^{26} i_{\Gamma}=6$ we have seemingly two operators, namely

$$
\begin{aligned}
& \mathcal{O}_{11}^{i_{\Gamma}=6}=g^{\alpha \beta} \mathcal{K}_{\alpha \mu_{1}} \cdot \mathcal{K}_{\mu_{2}} \cdot \mathcal{K}_{\mu_{3}} \cdot \mathcal{K}_{\mu_{4} \beta} E^{\mu_{1} \mu_{2} \mu_{3} \mu_{4}} \\
& \mathcal{O}_{12}^{i_{\Gamma}=6}=\left(\mathcal{K}_{\mu_{1}}: \mathcal{K}_{\mu_{2}}\right)\left(\mathcal{K}_{\mu_{3}}: \mathcal{K}_{\mu_{4}}\right) E^{\mu_{1} \mu_{2} \mu_{3} \mu_{4}}
\end{aligned}
$$

which however both vanish due to the symmetry (cyclic symmetry and $\mu_{1} \mu_{2}$ and $\mu_{3} \mu_{4}$ symmetry respectively) of the four times contracted extrinsic curvature tensors. In the case $n_{j}=1, m_{j}=3$, we need three contractions within the $D \mathcal{K}^{3}$ building block so we have only the following possibility

$$
\mathcal{O}_{13}^{i_{\Gamma}=6}=g^{\alpha \beta}\left(D_{\mu_{1}} \mathcal{K}_{\alpha \mu_{2}}\right) \cdot \mathcal{K}_{\mu_{3}} \cdot \mathcal{K}_{\mu_{4} \beta} E^{\mu_{1} \mu_{2} \mu_{3} \mu_{4}},
$$

which, however, vanish due to (A.2). For $n_{j}=2$ and $m_{j}=2$, we need two contractions within $D^{2} \mathcal{K}^{2}$, the only possibility with one uncontracted index per $\mathcal{K}$ is then (modulo integration by parts)

$$
\mathcal{O}_{14}^{i_{\Gamma}=6}=\left(D_{\mu_{1}} \mathcal{K}_{\mu_{2}}\right):\left(D_{\mu_{3}} \mathcal{K}_{\mu_{4}}\right) E^{\mu_{1} \mu_{2} \mu_{3} \mu_{4}}
$$

but this operator vanishes using (A.2). Finally, in the case $n_{j}=3$ and $m_{j}=1$, only one contraction is needed within $D^{3} \mathcal{K}$, however, because $\mathcal{K}_{\alpha \mu \nu}$ is totally symmetric and traceless, the subsequent contraction with $E^{\mu_{1} \mu_{2} \mu_{3} \mu_{4}}$ vanishes. To summarize, there is no parity odd on-shell operator up to and including $i_{\Gamma}=6$.

\section{Acknowledgments}

The authors thank Mariana Carrillo Gonzalez, Riccardo Penco and Mark Trodden for the correspondence. This work is supported in part by Czech Science Foundation (Project No. GAČR 18-17224S) and by Ministry of Education, Youth and Sports of the Czech Republic (Project No. LTAUSA17069).

\footnotetext{
${ }^{26}$ Note that the operators with $n_{j}+3 m_{j}$ odd cannot be constructed, therefore there are no $i_{\Gamma}=5$ parity odd operators.
} 
Open Access. This article is distributed under the terms of the Creative Commons Attribution License (CC-BY 4.0), which permits any use, distribution and reproduction in any medium, provided the original author(s) and source are credited.

\section{References}

[1] G.R. Dvali, G. Gabadadze and M. Porrati, $4-D$ gravity on a brane in $5-D$ Minkowski space, Phys. Lett. B 485 (2000) 208 [hep-th/0005016] [INSPIRE].

[2] C. Deffayet, G.R. Dvali and G. Gabadadze, Accelerated universe from gravity leaking to extra dimensions, Phys. Rev. D 65 (2002) 044023 [astro-ph/0105068] [INSPIRE].

[3] C. de Rham, G. Gabadadze and A.J. Tolley, Resummation of Massive Gravity, Phys. Rev. Lett. 106 (2011) 231101 [arXiv:1011.1232] [INSPIRE].

[4] A. Nicolis, R. Rattazzi and E. Trincherini, The Galileon as a local modification of gravity, Phys. Rev. D 79 (2009) 064036 [arXiv:0811.2197] [INSPIRE].

[5] A.I. Vainshtein, To the problem of nonvanishing gravitation mass, Phys. Lett. B 39 (1972) 393 [InSPIRE].

[6] M.A. Luty, M. Porrati and R. Rattazzi, Strong interactions and stability in the DGP model, JHEP 09 (2003) 029 [hep-th/0303116] [INSPIRE].

[7] K. Hinterbichler, M. Trodden and D. Wesley, Multi-field galileons and higher co-dimension branes, Phys. Rev. D 82 (2010) 124018 [arXiv: 1008.1305] [INSPIRE].

[8] C. de Rham, G. Gabadadze, L. Heisenberg and D. Pirtskhalava, Nonrenormalization and naturalness in a class of scalar-tensor theories, Phys. Rev. D 87 (2013) 085017 [arXiv: 1212.4128] [INSPIRE].

[9] C. Deffayet, S. Deser and G. Esposito-Farese, Generalized Galileons: All scalar models whose curved background extensions maintain second-order field equations and stress-tensors, Phys. Rev. D 80 (2009) 064015 [arXiv: 0906.1967] [INSPIRE].

[10] C. Deffayet, S. Deser and G. Esposito-Farese, Arbitrary p-form Galileons, Phys. Rev. D 82 (2010) 061501 [arXiv:1007.5278] [INSPIRE].

[11] T.L. Curtright and D.B. Fairlie, A Galileon Primer, arXiv:1212.6972 [InSPIRE].

[12] J. Khoury, Les Houches Lectures on Physics Beyond the Standard Model of Cosmology, arXiv: 1312.2006 [INSPIRE].

[13] C. Cheung, K. Kampf, J. Novotny and J. Trnka, Effective Field Theories from Soft Limits of Scattering Amplitudes, Phys. Rev. Lett. 114 (2015) 221602 [arXiv:1412.4095] [INSPIRE].

[14] C. Cheung, K. Kampf, J. Novotny, C.-H. Shen and J. Trnka, On-Shell Recursion Relations for Effective Field Theories, Phys. Rev. Lett. 116 (2016) 041601 [arXiv:1509.03309] [INSPIRE].

[15] C. Cheung, K. Kampf, J. Novotny, C.-H. Shen and J. Trnka, A Periodic Table of Effective Field Theories, JHEP 02 (2017) 020 [arXiv:1611.03137] [INSPIRE].

[16] C. de Rham, M. Fasiello and A.J. Tolley, Galileon Duality, Phys. Lett. B 733 (2014) 46 [arXiv:1308.2702] [INSPIRE].

[17] C. De Rham, L. Keltner and A.J. Tolley, Generalized galileon duality, Phys. Rev. D 90 (2014) 024050 [arXiv: 1403.3690] [INSPIRE]. 
[18] P. Creminelli, M. Serone, G. Trevisan and E. Trincherini, Inequivalence of Coset Constructions for Spacetime Symmetries, JHEP 02 (2015) 037 [arXiv: 1403.3095] [INSPIRE].

[19] K. Kampf and J. Novotny, Unification of Galileon Dualities, JHEP 10 (2014) 006 [arXiv: 1403.6813] [INSPIRE].

[20] K. Hinterbichler and A. Joyce, Hidden symmetry of the Galileon, Phys. Rev. D 92 (2015) 023503 [arXiv:1501.07600] [InSPIRE].

[21] J. Novotny, Geometry of special Galileons, Phys. Rev. D 95 (2017) 065019 [arXiv: 1612.01738] [INSPIRE].

[22] F. Cachazo, S. He and E.Y. Yuan, Scattering Equations and Matrices: From Einstein To Yang-Mills, DBI and NLSM, JHEP 07 (2015) 149 [arXiv: 1412.3479] [INSPIRE].

[23] J. Gasser and H. Leutwyler, Chiral Perturbation Theory to One Loop, Annals Phys. 158 (1984) 142 [inSPIRE].

[24] J. Gasser and H. Leutwyler, Chiral Perturbation Theory: Expansions in the Mass of the Strange Quark, Nucl. Phys. B 250 (1985) 465 [InSPIRE].

[25] S. Garcia-Saenz, J. Kang and R. Penco, Gauged Galileons, JHEP 07 (2019) 081 [arXiv: 1905.05190] [INSPIRE].

[26] M. Carrillo González, R. Penco and M. Trodden, Shift symmetries, soft limits and the double copy beyond leading order, arXiv:1908.07531 [INSPIRE].

[27] N. Brouzakis, A. Codello, N. Tetradis and O. Zanusso, Quantum corrections in Galileon theories, Phys. Rev. D 89 (2014) 125017 [arXiv:1310.0187] [InSPIRE].

[28] N. Brouzakis and N. Tetradis, Suppression of Quantum Corrections by Classical Backgrounds, Phys. Rev. D 89 (2014) 125004 [arXiv:1401.2775] [InSPIRE].

[29] I.D. Saltas and V. Vitagliano, Quantum corrections for the cubic Galileon in the covariant language, JCAP 05 (2017) 020 [arXiv: 1612.08953] [INSPIRE].

[30] I.D. Saltas and V. Vitagliano, Covariantly Quantum Galileon, Phys. Rev. D 95 (2017) 105002 [arXiv: 1611.07984] [INSPIRE].

[31] G. Goon, K. Hinterbichler, A. Joyce and M. Trodden, Aspects of Galileon Non-Renormalization, JHEP 11 (2016) 100 [arXiv:1606.02295] [INSPIRE].

[32] L. Heisenberg and C.F. Steinwachs, One-loop renormalization in Galileon effective field theory, arXiv: 1909.04662 [INSPIRE].

[33] D.G. Boulware and L.S. Brown, Tree Graphs and Classical Fields, Phys. Rev. 172 (1968) 1628 [inSPIRE].

[34] I. Ya. Arefeva, L.D. Faddeev and A.A. Slavnov, Generating Functional for the s Matrix in Gauge Theories, Theor. Math. Phys. 21 (1975) 1165 [Teor. Mat. Fiz. 21 (1974) 311] [INSPIRE].

[35] A. Jevicki and C.-k. Lee, The S Matrix Generating Functional and Effective Action, Phys. Rev. D 37 (1988) 1485 [INSPIRE].

[36] G. 't Hooft, An algorithm for the poles at dimension four in the dimensional regularization procedure, Nucl. Phys. B 62 (1973) 444 [INSPIRE]. 
[37] C.-k. Lee and C. Rim, Background Fermi Fields and Schwinger-dewitt Proper Time Method, Nucl. Phys. B 255 (1985) 439 [inSPIRE].

[38] B.S. DeWitt, The global approach to quantum field theory. Vol. 1, 2, Int. Ser. Monogr. Phys. 114 (2003) 1 [INSPIRE].

[39] D.V. Vassilevich, Heat kernel expansion: User's manual, Phys. Rept. 388 (2003) 279 [hep-th/0306138] [INSPIRE].

[40] M. Buchler and G. Colangelo, Renormalization group equations for effective field theories, Eur. Phys. J. C 32 (2003) 427 [hep-ph/0309049] [INSPIRE].

[41] H. Elvang, M. Hadjiantonis, C.R.T. Jones and S. Paranjape, Soft Bootstrap and Supersymmetry, JHEP 01 (2019) 195 [arXiv:1806.06079] [INSPIRE]. 